ESAIM: PROCEEDINGS, May 2009, Vol. 27, p. 180-208

C. Besse, O. Goubet, T. Goudon \& S. Nicaise, Editors

\title{
SÉPARATION DES ÉCHELLES ET SCHÉMAS MULTINIVEAUX POUR LES ÉQUATIONS D'ONDES NON-LINÉAIRES
}

\author{
C. Calgaro ${ }^{1}$, J.-P. Chehab ${ }^{2}$, J. Laminie ${ }^{3}$ And E. Zahrouni ${ }^{4}$
}

\begin{abstract}
In this work, we point out and apply some ideas for the numerical simulation by multilevel schemes of several wave equations including dispersive (Korteweg de Vries) and dissipative ones (Kuramoto-Sivashinski). We start from the approach developed for dissipative equations decomposing the solution into large $(\mathrm{Y})$ and small $(\mathrm{Z})$ scales, and we show that it is necessary to have a regularization in time to allow stable numerical treatment of $\mathrm{Y}$ and $\mathrm{Z}$ which are here generated using hierarchical processes such as Wavelets, Incremental Unknowns or Fourier.
\end{abstract}

Résumé. Nous proposons dans cet article de mettre en avant quelques idées pour la simulation numérique de plusieurs classes d'équations d'ondes dispersives (Korteweg de Vries) ou non (KuramotoSivashinski) par des méthodes multiniveaux. Nous partons des idées initialement développées pour les équations dissipatives et en proposons une extension. Il en ressort que la présence d'une régularisation en temps est nécessaire pour pouvoir traiter de manière stable, et avec des schémas appropriés, les différentes échelles de la solution, préalablement générées à l'aide de méthodes hiérarchiques (inconnues incrémentales, ondelettes, Fourier).

\section{INTRODUCTION}

La simulation numérique de systèmes dynamiques met souvent en évidence la présence d'échelles (ou de structures) de tailles différentes ; on note canoniquement $Y$ les grandes structures et $Z$ les petites structures. Les $Y$ sont associés à la partie principale de la solution, les $Z$ à une partie fluctuante. Dans la simulation de la turbulence, les méthodes LES ou de sous-mailles proposent de traiter ces structures numériquement de maniére différente, en s'appuyant par exemple sur une modélisation (relation de fermeture). Au milieu des années 80, et pour des systèmes dynamiques dissipatifs, les travaux de Temam et al [Tem97] ont permis de justifier mathématiquement la démarche de séparation des échelles (théorie des variétés inertielles). D'abord formulés dans un cadre spectral, les méthodes de Galerkin non-linéaires (GNL) ont été présentées comme un cadre numérique pour mettre en application la modélisation des $Z$ par les $Y$. La méthode a été étendue par la suite à des situations plus générales, citons Marion-Temam [MT89, MT90] pour les éléments finis, Temam [CT91a, CT91b] pour les différences finies, Goubet [Gou93] pour les ondelettes.

\footnotetext{
${ }^{1}$ Laboratoire Paul Painlevé, UMR 8524, Université de Lille 1, France, (caterina.calgaro@univ-lille1.fr) and EPI SIMPAF INRIA Lille Nord Europe

${ }^{2}$ LAMFA, UMR 6140, Université de Picardie Jules Verne, Amiens, France (jean-paul.chehab@u-picardie.fr) and EPI SIMPAF INRIA Lille Nord Europe

${ }^{3}$ Grimaag-Guadeloupe Campus de Fouillole B.P. 59297157 Pointe à Pitre Cedex (jacques.laminie@math.u-psud.fr) and Laboratoire de Mathématique, Université Paris 11, Orsay, France

4 Département de Mathématiques, Faculté des Sciences de Monastir, Boulevard de l'Environnement, 5000 Monastir, Tunisie
}

(C) EDP Sciences, SMAI 2009 
Dans la pratique, la projection sur une variété inertielle (approchée) ne permet pas de mettre en avant l'efficacité de la démarche en termes de réduction de temps de calcul, comme l'a montré F. Pascal [Pas92] en éléments finis.

En fait, l'idée principale de la démarche consiste à traiter différemment les $Y$ et les $Z$, c'est-à-dire à définir un schéma numérique différent pour les $Y$ et pour les $Z$. Cette approche a donné lieu à de nouveaux types de schémas "multiniveaux en temps" : citons par exemple Debussche-Dubois-Temam [DDT95] et DuboisJauberteau-Temam [DJT98] en spectral pour la simulation de la turbulence homogène, Calgaro-DebusscheLaminie-Temam [CLT97, CDL98, CL08] en éléments finis (hiérarchiques) pour les équations de Burgers et de Navier-Stokes, Costa-Dettori-Gottlieb-Temam [CDGT01] pour Burgers en Fourier et Chebyshev, Pouit [Pou98] et Chehab-Costa [CC03, CC04] en différences finies pour Burgers, Debussche-Laminie-Zahrouni [DLZ05] en ondelettes et éléments finis pour Burgers.

Tous les travaux sus-cités s'appuyent sur une décomposition a priori des inconnues en termes de grandes et de petites structures - les techniques utilisées à cet effet varient suivant le contexte, nous y reviendrons plus tard - et sur un traitement numérique différencié de celles-ci. Cette stratégie se justifie par un phénomène observé dans certains systèmes dissipatifs, à savoir le transfert de l'énergie de la solution des hauts vers les bas modes de Fourier, ce qui peut se voir également comme une propriété de régularisation [Tem97].

Dans cet article, nous appliquons cette approche à des équations d'ondes dispersives ou non et qui présentent des caractéristiques toutes autres. En particulier, certaines d'entre elles admettent des solutions localisées en espace (solitons) ou peuvent présenter des phénomènes d'explosion en temps fini ; dans ce dernier cas les transferts d'énergie des hauts modes vers les bas modes ne peuvent avoir lieu.

Nous considérons ici successivement quelques équations d'ondes 1D pour lesquelles nous proposons des schémas multiniveaux s'appuyant sur une décomposition de type Y-Z, en Fourier, en inconnues incrémentales en différences finies et en ondelettes interpolantes suivant les cas ; l'utilisation des ondelettes interpolantes est motivée pour le développement de maillages adaptatifs et le calcul de solutions, explosives ou non, très localisées en espace. Bien que l'implémentation des schémas numériques proposés ne soit réalisée qu'en considérant deux niveaux de grilles, elle permet déjà d'explorer et de conclure sur l'efficacité ou non des techniques multiniveaux étudiées.

Notre travail est organisé comme suit : dans un premier temps nous rappelons la discrétisation spatiale sur une grille dyadique et quelques décompositions en espace / fréquences (inconnues incrémentales et ondelettes interpolantes) que nous utiliserons pour définir les différents niveaux d'échelles. Ensuite, pour un problème modèle assez général, nous proposons des schémas de discrétisation en temps vérifiant des propriétés de conservation d'invariants au niveau discret. Puis, pour l'équation de Korteweg de Vries (KdV), nous introduisons différents schémas multiniveaux de type Crank-Nicolson ou Runge Kutta. L'approche de type GNL n'est pas concluante dans ce cas ; en revanche nous proposons quelques pistes de résolutions adaptatives avec des techniques de seuillage. L'équation de Kuramoto-Sivashinski qui comporte des termes dissipatifs, présente en revanche des propriétés de régularisation, ce qui permet d'appliquer avec succès les techniques multiniveaux de type GNL. Des propriétés de régularisation semblent donc être un préalable à l'application d'une telle approche multiniveaux et il serait à cet égard intéressant de considérer les équations faiblement amorties, telles que celles de KdV faiblement amortie [AMCC08, Ghi88, Ghi94, Gou00, GR02].

\section{Discrétisation en espace et SÉparation Des ÉCHelles}

Considérons dans l'équation d'évolution non linéaire suivante, posée dans un espace de Hilbert $H$ :

$$
\left\{\begin{aligned}
u_{t} & =L(t, u)+N(t, u), & & x \in] 0, \ell[, \quad t>0 \\
u(0, x) & =u_{0}(x), & & x \in] 0, \ell[
\end{aligned}\right.
$$

où $L$ (resp. $N$ ) est un opérateur linéaire (resp. non linéaire). Le problème de Cauchy (2.1) sera complété par des conditions aux limites ; dans la suite de l'article on les considérera périodiques, mais ceci seulement pour simplifier la présentation des méthodes de séparation des variables (ondelettes et inconnues incrémentales). 
Afin de discrétiser en espace le problème (2.1) on considère, pour tout entier $j \geq 0$, la grille formée des points dyadiques

$$
\mathcal{G}_{j}=\left\{x_{j, k}=\frac{k \ell}{2^{j}} \text { pour } k=0, \ldots, 2^{j}-1\right\} .
$$

$\mathcal{G}_{j}$, dite grille de niveau $j$, réalise une subdivision équidistante de $[0, \ell[$. On considère une solution $u=u(t, x)$ de (2.1) que l'on suppose assez régulière de manière à ce qu'il soit possible d'approcher, pour tout $t \geq 0$ et sur chaque grille $\mathcal{G}_{j}, j \geq 0$, la solution $u(t, x)$ aux points $x_{j, k}$ par les valeurs $U_{j}(t) \in \mathbb{R}^{2^{j}}$. La fonction vectorielle $U_{j}(t)$ est alors définie comme solution d'un système différentiel de la forme

$$
\left\{\begin{array}{l}
\frac{d}{d t} U_{j}(t)=L^{(j)}\left(t, U_{j}\right)+N^{(j)}\left(t, U_{j}\right) \\
U_{j}(0)=U_{0, j}
\end{array}\right.
$$

Le système (2.3) est obtenu en appliquant une méthode de différences finies d'ordre $p>0$ pair. Ici $L^{(j)}$ et $N^{(j)}$ sont les opérateurs $L$ et $N$ discrétisés sur la grille de niveau $j$.

\subsection{La décomposition en grandes et petites échelles}

La simulation numérique de systèmes dynamiques met souvent en évidence la présence d'échelles (ou de structures) de tailles différentes, associées respectivement aux modes lents et modes rapides du flot. D'autre part, la stabilité des schémas numériques classiques est subordonnée à leur capacité à représenter les fréquences élevées de la solution. Ces schémas reposent sur un traitement uniforme de toutes les données ; la stabilité numérique est donc limitée par le comportement des hautes fréquences par rapport à la discrétisation considérée.

Un moyen d'augmenter la stabilité des schémas numériques et donc de pouvoir calculer plus précisement, mais aussi plus rapidement, la solution du problème pour les grands intervalles de temps consiste à introduire une décomposition des inconnues en termes de grandes et petites structures et de traiter celles-ci différemment dans le schéma numérique en temps. Plus précisement, la décomposition en grandes et petites structures consiste à réorganiser a priori les données en termes de partie principale et partie fluctuante de la solution. Il est à noter que cette décomposition diffère des décompositions LES (Large Eddies Simulations) $u=\bar{u}+\tilde{u}$ dans lesquelles la partie principale $\bar{u}$ est bien de l'ordre de la solution physique $u$ mais la partie fluctuante $\tilde{u}$, petite en termes d'énergie et fluctuante en temps, n'est pas représentée dans la simulation. Dans la décomposition que nous considérerons, la partie fluctuante, petite en espace, sera représentée dans la simulation.

Lorsque les conditions aux limites sont périodiques, on approche pour tout $t \geq 0$ les fonctions assez régulières par des séries de Fourier tronquées :

$$
u(t, x) \simeq \sum_{i=1}^{2 n} \alpha_{i}(t) w_{i}(x)
$$

où les $\alpha_{i}(t)$ sont des réels et les $w_{i}(x)$ les $2 n$ premiers éléments d'une base hilbertienne de $H$. Nous avons alors évidemment

$$
u(t, x)=\sum_{i=1}^{n} \alpha_{i}(t) w_{i}(x)+\sum_{i+1}^{2 n} \alpha_{i}(t) w_{i}(x)=y(t, x)+z(t, x) .
$$

Pour tout $t$, on pose $Y(t)=\left(\alpha_{1}(t), \ldots, \alpha_{n}(t)\right)^{T}$ et $Z(t)=\left(\alpha_{n+1}(t), \ldots, \alpha_{2 n}(t)\right)^{T}$. Le vecteur $Y$ est constitué des les petites longueurs d'ondes et, de par la convergence de la série, contient la majeure partie de l'énergie. Le vecteur $Z$ est associé aux fréquences élevées et ses composantes sont "petites" par rapport à celles de $Y$. La mise en œuvre de cette décomposition s'effectue au moyen de FFT. Néanmoins, cette décomposition a deux inconvénients : d'une part, elle ne s'applique qu'aux conditions aux limites périodiques et, d'autre part, les différentes échelles ne sont pas du tout localisées en espace, ce qui oblige à effectuer des calculs coûteux pour capter des phénomènes très localisés : cette décomposition est donc parfaitement localisée en fréquence mais pas du tout en espace. 
Dans le cas non périodique, ou si on veut travailler localement en espace, les méthodes hiérarchiques offrent un compromis très intéressant pour séparer les échelles à la fois en fréquence et en espace. Ces méthodes (inconnues incrémentales ou ondelettes en différences finies, bases hiérarchiques en éléments finis,...) reposent toutes sur le même schéma de construction. Tout d'abord, on considère plusieurs grilles de discrétisation en espace et l'on distingue les inconnues portées par la grille grossière de celles portées par les grilles complémentaires : la grille grossière ne peut représenter que les basses fréquences alors que les grilles fines complémentaires peuvent capter les modes élevés, en réalisant une séparation en fréquence de la solution. Dans un deuxième temps, on remplace les inconnues de grilles fines complémentaires par des nouvelles inconnues qui représentent localement l'erreur d'interpolation. On opère ainsi une séparation des échelles en espace. Nous détaillons dans la suite les décompositions en ondelettes et en différences finies.

Si l'on omet pour l'instant un éventuel seuillage numérique, cette décomposition consiste en un changement de variables (linéaire) que l'on représente par une matrice de transfert $S$; la transformation est en effet linéaire en l'absence de seuillage. La matrice $S$ représente aussi bien une FFT, une transformée en ondelettes interpolantes, qu'une matrice de tranfert de bases hiérarchiques ou d'inconnues incrémentales.

Plus précisément, si à chaque instant $t$, le vecteur $U(t)$ contient les approximations nodales de la solution, on définit $\hat{U}(t)$ par la relation

$$
U(t)=S \hat{U}(t)
$$

avec $\hat{U}(t)=(Y(t), Z(t))^{T}$. Ici, $Y(t)$ correspond à l'approximation de la solution dans l'espace grossier, $Y(t)$ est donc de l'ordre de la solution physique à l'instant $t$, tandis que $Z(t)$ contient les corrections à la solution correspondants aux erreurs d'interpolation sur une ou plusieurs grilles fines successives.

\subsection{Interpolation de Lagrange et compression des données}

Afin de simplifier la présentation, on travaillera d'abord sur la droite réelle ; il sera simple de se restreindre ensuite aux fonctions périodiques sur l'intervalle $[0, \ell]$. Pour tout entier $j \geq 0$, la grille de niveau $j$ qui réalise une subdivision équidistante de $\mathbb{R}$ sera encore notée

$$
\mathcal{G}_{j}=\left\{x_{j, k}=\frac{k}{2^{j}} \text { pour } k \in \mathbb{Z}\right\}
$$

et on a

$$
\begin{gathered}
\mathcal{G}_{j} \subset \mathcal{G}_{j+1}, \\
\mathcal{G}_{j+1} \backslash \mathcal{G}_{j}=\left\{x_{j+1,2 k+1}=\frac{2 k+1}{2^{j+1}} \text { pour } k \in \mathbb{Z}\right\} .
\end{gathered}
$$

On remarque qu'un nœud de $\mathcal{G}_{j+1}$ est soit un nœud de $\mathcal{G}_{j}$ soit le milieu de deux nœuds successifs de $\mathcal{G}_{j}$.

En suivant [DD89], nous rappelons le schéma de subdivision qui nous servira dans la suite pour réaliser les opérations de décomposition (ou d'analyse) et de synthèse de la solution d'un problème aux limites. Soit $f$ une fonction continue définie sur $\mathbb{R}$ et soit $\left(f_{j, k}\right)_{k}$ la suite définie par $f_{j, k}=f\left(x_{j, k}\right)$ pour $k \in \mathbb{Z}$. Etant donné un entier $M$ pair, on construit la suite $\left(f_{j+1, k}\right)_{k}$ approchant $f$ aux points de $\mathcal{G}_{j+1}$ par le schéma suivant :

$$
f_{j+1,2 k}=f_{j, k}, \quad f_{j+1,2 k+1}=P_{j+1,2 k+1}\left(x_{j+1,2 k+1}\right),
$$

où $P_{j+1,2 k+1}$ est le polynôme d'interpolation de Lagrange d'ordre $M-1$ défini par

$$
-\frac{M}{2}+1 \leq l \leq \frac{M}{2}, \quad P_{j+1,2 k+1}\left(x_{j, k+l}\right)=f_{j, k+l} .
$$


Le polynôme $P_{j+1,2 k+1}$ nous permet d'evaluer les valeurs de $f$ aux points de $\mathcal{G}_{j+1} \backslash \mathcal{G}_{j}$ par interpolation des valeurs approchées de $f$ sur la grille $\mathcal{G}_{j}$. D'après $(2.5)$, on a alors

$$
f_{j+1,2 k+1}=\sum_{l=-\frac{M}{2}+1}^{\frac{M}{2}} h_{l} f_{j, k+l} .
$$

Puisque les points de $\mathcal{G}_{j}$ sont équidistants, les coefficients $h_{l}$ vérifient :

$$
h_{l}=(-1)^{\frac{M}{2}+l-1} \frac{\prod_{i=0}^{M-1}\left(i-\frac{M}{2}+\frac{1}{2}\right)}{\left(l-\frac{1}{2}\right)\left(\frac{M}{2}+l-1\right) !\left(\frac{M}{2}-l\right) !} \quad \text { pour }-\frac{M}{2}+1 \leq l \leq \frac{M}{2} .
$$

\subsubsection{Ondelettes interpolantes et analyse multirésolution}

La solution du problème modèle (2.1) peut être approchée par des fonctions appartenant à des sous espaces de dimension finie engendrés par des fonctions périodiques, réguliéres et polynômiales par morceaux. Ces fonctions sont générées par des opérations de dilatation et de translation de la périodisée d'une unique fonction régulière $\varphi$ à support compact, dite fonction d'échelle.

La fonction $\varphi$ va être construite de proche en proche sur chaque grille $\mathcal{G}_{j}$. Pour $j=0$, on considère une fonction $\varphi$ qui interpole la suite de Kronecker pour tout entier $k: \varphi(k)=\delta_{k, 0}$, où $\delta_{k, j}$ désigne le symbole de Kronecker. Pour tout $j$, les valeurs de $\varphi$ sur $\mathcal{G}_{j+1}$ sont données par

$$
\forall j \geq 0, \forall k \in \mathbb{Z}, \quad \varphi\left(x_{j+1,2 k+1}\right)=\sum_{l=-\frac{M}{2}+1}^{\frac{M}{2}} h_{l} \varphi\left(x_{j, k+l}\right),
$$

avec $h_{l}$ défini par (2.7). La fonction $\varphi$ ainsi construite sur les rationnels s'étend d'une manière unique par continuité à $x \in \mathbb{R}$. En outre, $\varphi$ est une fonction d'échelle interpolante, à support compact,

$$
\operatorname{supp}(\varphi)=[-M+1, M-1]
$$

vérifiant une relation à deux échelles,

$$
\varphi(x)=\sum_{r=-M+1}^{M-1} g_{r} \varphi(2 x-r) .
$$

Les coefficients $g_{r}$ sont donnés à partir des coefficients $h_{r}$ définis par (2.7),

$$
g_{r}=\left\{\begin{array}{cccc}
1 & r=0, & \\
0 & r \neq 0 & r & \text { est pair } \\
h_{\frac{r+1}{2}} & & r & \text { est impair. }
\end{array}\right.
$$

Enfin, tout polynôme de degré inférieur ou égal à $M-1$ est obtenu comme combinaison linéaire de la famille de fonctions $\varphi(x-k), k \in \mathbb{Z}$. Pour la régularité Hölderienne de $\varphi$ voir [DD89] et [Dau93].

On pose

$$
\forall j \geq 0, \forall k \in \mathbb{Z}, \quad \varphi_{j, k}(x)=\varphi\left(2^{j} x-k\right)
$$

On remarque que

$$
\forall k, k^{\prime} \in \mathbb{Z}, \quad \varphi_{j, k}\left(x_{j, k^{\prime}}\right)=\delta_{k, k^{\prime}}
$$


La famille $\left(\varphi_{j, k}\right)_{k}$ est dite alors interpolante sur la grille $\mathcal{G}_{j}$. A la fonction d'échelle $\varphi$ est associée la fonction

$$
\psi(x)=\varphi(2 x-1)
$$

qui est appelée fonction ondelette. On pose

$$
\forall j \geq 0, \forall k \in \mathbb{Z}, \quad \psi_{j, k}(x)=\psi\left(2^{j} x-k\right),
$$

alors

$$
\forall k, k^{\prime} \in \mathbb{Z}, \quad \psi_{j, k}\left(x_{j, k^{\prime}}\right)=0 \quad \text { et } \quad \psi_{j, k}\left(x_{j+1,2 k^{\prime}+1}\right)=\delta_{k, k^{\prime}}
$$

En suivant [Don92], on introduit pour tout $j \geq 0$ les suites de sous-espaces vectoriels de $L^{2}(\mathbb{R})$

$$
\begin{aligned}
V_{j} & ={\overline{\operatorname{Vect}}\left\{\varphi_{j, k}, k \in \mathbb{Z}\right\}}^{L^{2}}, \\
W_{j} & =\overline{\operatorname{Vect}\left\{\psi_{j, k}, k \in \mathbb{Z}\right\}}{ }^{2} .
\end{aligned}
$$

Grâce à (2.10) et (2.12), on a :

$$
V_{j} \subset V_{j+1}, \quad V_{j+1}=V_{j} \oplus W_{j}, \quad \forall j \geq 0 .
$$

Les fonctions $\varphi$ et $\psi$ ne vérifient pas les conditions aux limites périodiques sur l'intervalle $[0, \ell]$. Afin de construire une base d'ondelettes interpolantes et périodiques sur $[0, \ell]$, on commence par construire une base d'ondelettes de $L^{2}([0,1])$, en périodisant chaque ondelette $\psi_{j, k}$ et chaque fonction d'échelle $\varphi_{j, k}$. Puis, une base d'ondelettes de $L^{2}([0, \ell])$ se construit par une dilatation d'un facteur $\ell$ des ondelettes de la base de $L^{2}([0,1])$. Pour les détails sur la construction des ondelettes de bord voir [Mal00] et ses références.

Par périodisation sur $[0,1]$, on a $\forall j \geq 0$ et $k \in\left\{0, \ldots, 2^{j}-1\right\}$ les fonctions suivantes :

$$
\begin{aligned}
& \varphi_{j, k}^{\text {per }}(x)=\sum_{m \in \mathbb{Z}} \varphi_{j, k}(x-m), \\
& \psi_{j, k}^{\text {per }}(x)=\sum_{m \in \mathbb{Z}} \psi_{j, k}(x-m) .
\end{aligned}
$$

On vérifie alors que

$$
\varphi_{j, k}^{p e r}(x+1)=\varphi_{j, k}^{p e r}(x) \quad \text { et } \quad \varphi_{j, k+2^{j}}^{p e r}(x)=\varphi_{j, k}^{p e r}(x) .
$$

Il en est de même pour les ondelettes $\psi_{j, k}^{\text {per }}$. De plus, les fonctions $\varphi_{j, k}^{\text {per }}$ vérifient l'équation de dilatation (2.10) et sont interpolantes sur $\mathcal{G}_{j}$ tandis que les fonctions $\psi_{j, k}^{p e r}$ s'annulent aux points $\mathcal{G}_{j}$ et sont interpolantes sur $\mathcal{G}_{j+1} \backslash \mathcal{G}_{j}$. On considère alors les sous espaces de $L^{2}([0,1])$

$$
\begin{gathered}
V_{j}^{\text {per }}=\operatorname{Vect}\left\{\varphi_{j, k}^{\text {per }}, k \in\left\{0, \ldots, 2^{j}-1\right\}\right\}, \\
W_{j}^{\text {per }}=\operatorname{Vect}\left\{\psi_{j, k}^{\text {per }}, k \in\left\{1, \ldots, 2^{j}\right\}\right\} .
\end{gathered}
$$

On remarque que $V_{j}^{\text {per }}$ et $W_{j}^{\text {per }}$ sont des sous-espaces de dimension $2^{j}$.

Finalement, nous présentons les algorithmes d'analyse et de synthèse de la solution $u(t, x)$ du problème modèle (2.1) à chaque instant $t \geq 0$. On continuera à utiliser les mêmes notations pour les fonctions périodiques 
sur l'intervalle $[0, \ell]$, en remarquant que pour tout $j \geq 0,(2.2)$ sera la grille de niveau $j$. Si pour tout $t$ $u(t, x) \in V_{j+1}^{p e r}$, alors on a d'une part,

$$
u(t, x)=\sum_{k=0}^{2^{j+1}-1} \alpha_{j+1, k}(t) \varphi_{j+1, k}^{p e r}(x)
$$

et d'autre part,

$$
\begin{aligned}
u(t, x) & =y(t, x)+z(t, x), \\
y(t, x) & =\sum_{k=0}^{2^{j}-1} \alpha_{j, k}(t) \varphi_{j, k}^{p e r}(x), \\
z(t, x) & =\sum_{k=1}^{2^{j}} \beta_{j, k}(t) \psi_{j, k}^{\text {per }}(x) .
\end{aligned}
$$

De plus,

$$
u\left(t, x_{j, k}\right)=\alpha_{j+1,2 k}(t)=\alpha_{j, k}(t)
$$

car les fonctions $\psi_{j, k}^{p e r}$ s'annulent aux points de la forme $x_{j+1,2 k}$, et

$$
u\left(t, x_{j+1,2 k+1}\right)=y\left(t, x_{j+1,2 k+1}\right)+z\left(t, x_{j+1,2 k+1}\right)=\alpha_{j+1,2 k+1}(t) .
$$

Or la famille $\psi_{j, k}^{\text {per }}$ est interpolante aux points $x_{j+1,2 k+1}$ de la grille $\mathcal{G}_{j+1}$, donc

$$
z\left(t, x_{j+1,2 k+1}\right)=\beta_{j, k}(t)
$$

Grâce à (2.6), on a

et finalement les relations

$$
y\left(t, x_{j+1,2 k+1}\right)=\sum_{l=-\frac{M}{2}+1}^{\frac{M}{2}} h_{l} \alpha_{j, k+l}
$$

$$
\begin{aligned}
& \alpha_{j, k}(t)=\alpha_{j+1,2 k}(t), \\
& \beta_{j, k}(t)=\alpha_{j+1,2 k+1}(t)-\sum_{l=-\frac{M}{2}+1}^{\frac{M}{2}} h_{l} \alpha_{j, k+l}(t) .
\end{aligned}
$$

La séparation des échelles est ainsi réalisée, les $y$ sont de l'ordre de la solution physique tandis que les $z$ sont des détails portés par l'espace complémentaire.

Remarque 1. Puisque $V_{j+1}^{\text {per }}=V_{j}^{\text {per }} \oplus W_{j}^{\text {per }}$ pour chaque $j \geq 0$, alors de façon récursive $V_{j+1}^{\text {per }}=V_{j_{0}}^{\text {per }} \oplus W_{j_{0}}^{\text {per }} \oplus$ $\cdots \oplus W_{j}^{\text {per }}$ pour tout $j \geq j_{0} \geq 0$. L'analyse de la solution $u(t, x) \in V_{j+1}^{\text {per }}$ peut donc se réaliser récursivement sur plusieurs grilles afin de faire apparaître des détails de la solution de tailles différentes. La décomposition des sous-espaces de $L^{2}([0, \ell])$ permet alors d'écrire $u(t, x)=y_{j_{0}}(t, x)+z_{j_{0}}(t, x)+\cdots+z_{j}(t, x)$. Les valeurs de ces fonctions aux points des grilles dyadiques successives s'obtiennent récursivement par les relations (2.15).

\subsubsection{Différences finies et inconnues incrémentales}

A chaque instant $t$ et pour tout $j \geq 0$, soit $U_{j+1}(t)$ le vecteur de $\mathbb{R}^{2^{j+1}}$ correspondant à l'approximation nodale sur la grille $\mathcal{G}_{j+1}$ de la solution de (2.3) obtenue par l'application d'une méthode des différences finies d'ordre $p$. La construction des inconnues incrémentales (II) s'effectue en deux étapes : (1) hiérarchisation des inconnues, (2) changement de variables (récursif). Soit $j_{0}$ (resp. $J$ ) le niveau de discrétisation le plus grossier (resp. le plus fin) du domaine $\left[0, \ell\left[\right.\right.$. La hiérarchisation est une permutation $P_{\sigma}$ qui consiste à ranger d'abord les inconnues 
relatives à la grille grossière $\mathcal{G}_{j_{0}}$ puis celles des grilles complémentaires successives $\mathcal{G}_{j_{0}+1} \backslash \mathcal{G}_{j_{0}}, \ldots, \mathcal{G}_{J} \backslash \mathcal{G}_{J-1}$. Le changement de variables entre deux grilles $j$ et $j+1$ consiste à laisser inchangées les inconnues de la grille grossière $\mathcal{G}_{j}$ et à remplacer celles de la grille complémentaire $\mathcal{G}_{j+1} \backslash \mathcal{G}_{j}$ par une erreur d'interpolation d'ordre $M$. On pose alors $\hat{U}_{j+1}(t)=\left(Y_{j}(t), Z_{j}(t)\right)^{T}$ avec

$$
\begin{aligned}
& Y_{j, k}(t)=U_{j+1,2 k}(t)=U_{j, k}(t), \\
& Z_{j, k}(t)=U_{j+1,2 k+1}(t)-\sum_{l=-\frac{M}{2}+1}^{\frac{M}{2}} h_{l} U_{j, k+l}(t),
\end{aligned}
$$

la $k$-ième composante des vecteurs de $\mathbb{R}^{2^{j}}$. On parlera alors d'inconnues incrémentales d'ordre $M$. Les coefficients de la matrice $S=S_{j+1}$, dite matrice de transfert entre les grilles de niveau $j$ et $j+1$, sont déduits à partir des relations (2.16). En particulier, on peut écrire

$$
S_{j+1}=\left(\begin{array}{cc}
I d_{j} & 0 \\
R_{j} & I d_{j}
\end{array}\right)
$$

avec $I d_{j}$ la matrice identité de taille $2^{j}$ et $R_{j}$ une matrice creuse qui contient dans chaque ligne seulement $M$ coefficients non nuls (les coefficients $h_{l}$, voir $(2.7)$ ). On obtient alors

$$
P_{\sigma} U_{j+1}(t)=S_{j+1} \hat{U}_{j+1}(t)
$$

Quand plusieurs niveaux de grilles sont considérés, le changement de variables consiste à produire récursivement plusieurs blocs d'inconnues incrémentales. La matrice de transfert globale sera obtenue comme produit des matrices de transfert entre grilles successives :

$$
S_{j_{0}, J}=S_{J} S_{J-1} \cdots S_{j_{0}+1}
$$

et on a la relation $P_{\sigma} U_{J}(t)=S_{j_{0}, J} \hat{U}_{J}(t)$, avec $\hat{U}_{J}(t)=\left(Y_{j_{0}}(t), Z_{j_{0}}(t), \cdots, Z_{J-1}(t)\right)^{T}$.

Afin d'obtenir une meilleure compression des données avec des détails $Z$ de petite taille, l'ordre des opérateurs d'interpolation doit être élevé. Plus en général, si l'on considère des conditions aux limites autres que celles périodiques, cela se traduit dans la prise en compte d'un nombre important de valeurs pour interpoler les inconnues proches du bord. L'utilisation des schémas compacts (voir [Che98, Lel92]) permet d'atteindre une précision proche de celle du spectral en utilisant des différences finies et en prenant en compte des conditions aux limites générales.

Les schémas compacts sont des schémas implicites qui approchent un opérateur linéaire par une fraction rationnelle. Soit $f$ fonction régulière sur $[0, \ell]$; lorsque deux niveaux de discrétisation sont considérés, les valeurs interpolées aux points de la grille complémentaire $\mathcal{G}_{j+1} \backslash \mathcal{G}_{j}$ à partir des valeurs approchées sur la grille $\mathcal{G}_{j}$ sont définies par la relation

$$
\begin{aligned}
& f_{j+1,2 k+1}+\alpha\left(f_{j+1,2 k+3}+f_{j+1,2 k-1}\right)+\beta\left(f_{j+1,2 k+5}+f_{j+1,2 k-3}\right) \\
& =a\left(f_{j, k}+f_{j, k+1}\right)+b\left(f_{j, k-1}+f_{j, k+2}\right)+c\left(f_{j, k-2}+f_{j, k+3}\right), \quad k \in\left\{0, \ldots, 2^{j}-1\right\} .
\end{aligned}
$$

Ce système est fermé par les conditions aux limites périodiques. Les coefficients $\alpha, \beta, a, b, c$ sont déterminés en utilisant des développements de Taylor, de sorte à ce que l'identité (2.18) soit vérifiée à un ordre prescrit. Dès que $\alpha \neq 0$, le schéma est implicite ; par exemple pour $\alpha=\frac{1}{6}, a=\frac{2}{3}$ et $\beta=b=c=0$, on obtient un schéma d'interpolation d'ordre 4 . Le schéma d'interpolation se réécrit de manière générale sous la forme matricielle

$$
R_{j}=P^{-1} Q
$$


avec $P$ une matrice bande tri ou penta diagonale et $Q$ une matrice correspondante à la discrétisation de l'opérateur d'interpolation d'un ordre inférieur. La matrice de transfert entre les deux grilles est donnée par (2.17). Comme proposé dans [Che98], on peut adapter la construction des inconnues incrémentales aux conditions aux limites sous-jacentes au problème considéré, de sorte à s'affranchir d'effets de bord. On peut envisager d'autres conditions aux limites que celles périodiques, comme par exemple des conditions de Dirichlet homogènes ou des conditions de Neumann homogènes. Ces constructions s'étendent aussi à des grilles non régulières de type Tchebychev (voir [CM98]).

A titre d'illustration, nous comparons en Figure 1 la compression des données sur plusieurs niveaux d'inconnues incrémentales de la fonction $f(x)=\sin (90 x(1-x)) \sin \left(e^{3 x}\right)$ : les structures sont davantage localisées avec l'interpolation d'ordre élevé et sont plus petites en ordre de grandeur.
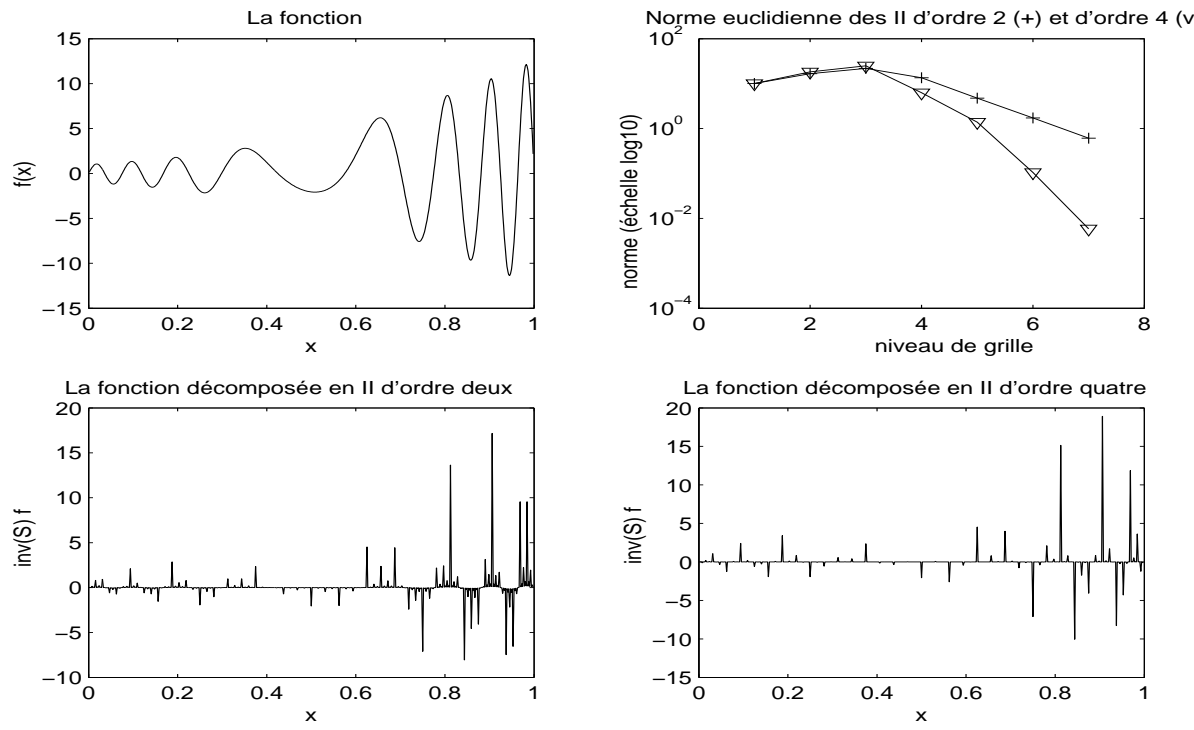

Figure 1. Compression des données en dimension un obtenue grâce aux inconnues incrémentales (II).

\subsection{Système différentiel à deux échelles}

Pour deux niveaux de grilles, le système différentiel (2.3) obtenu sur la grille fine de niveau $j+1$ peut se réecrire sous la forme d'un système couplé sur la grille grossière et sur la grille complémentaire :

$$
\begin{aligned}
& \frac{d Y_{j}}{d t}=L_{11}^{(j+1)} Y_{j}+L_{12}^{(j+1)} Z_{j}+N_{Y}^{(j+1)}\left(Y_{j}, Z_{j}\right), \\
& \frac{d Z_{j}}{d t}=L_{21}^{(j+1)} Y_{j}+L_{22}^{(j+1)} Z_{j}+N_{Z}^{(j+1)}\left(Y_{j}, Z_{j}\right) .
\end{aligned}
$$

Ici $L_{i j}^{(j+1)}$ désignent les blocs de la matrice $S_{j+1}^{-1} L^{(j+1)} S_{j+1}$ suivant la décomposition du vecteur d'inconnues $U_{j+1}$ en $Y_{j}$ et $Z_{j}$ :

$$
S_{j+1}^{-1} L^{(j+1)} S_{j+1}=\left(\begin{array}{cc}
L_{11}^{(j+1)} & L_{12}^{(j+1)} \\
L_{21}^{(j+1)} & L_{22}^{(j+1)}
\end{array}\right), \quad S^{-1} N^{(j+1)}\left(U_{j+1}\right)=\left(\begin{array}{c}
N_{Y}^{(j+1)}\left(Y_{j}, Z_{j}\right) \\
N_{Z}^{(j+1)}\left(Y_{j}, Z_{j}\right)
\end{array}\right) .
$$

En considérant plusieurs niveaux de détails de la solution et donc plusieurs niveaux de grilles $j_{0}, \ldots, j+$ $1=J$, la façon récursive de décomposer la solution sur les différentes grilles permet de réécrire le système 
différentiel (2.19) sous la forme d'un système couplé sur les grilles de niveau inférieur. Cela se fait exactement de façon analogue au passage du système différentiel (2.3) à celui couplé (2.19)-(2.20). Bien évidemment, le système différentiel relatif aux composantes $Y$ de la solution contiendra des termes linéaires et non-linéaires de couplage avec les différents niveaux de détails $Z$ de la solution.

Puisque dans la suite de l'article on considérera seulement deux niveaux de grilles, on simplifie les notations en écrivant $L^{(h)}$ et $N^{(h)}$ (resp. $L_{i j}^{(h)}$ et $N_{x}^{(h)}$ ) les discrétisations des opérateurs $L$ et $N$ sur la grille fine (resp. grilles grossière et complémentaire). De même, on notera $I d$ (resp. $I d_{1}, I d_{2}$ ) la matrice identité de la même taille que la matrice $L^{(h)}\left(\right.$ resp. $\left.L_{11}^{(h)}, L_{22}^{(h)}\right)$.

\section{Discrétisation En temps ET INVARIANTS}

Avant de détailler la semi-discrétisation en temps pour le problème (2.1), on rappelle le résultat formel suivant

Lemme 1. Soit $F: \mathbb{R} \rightarrow \mathbb{R}$ une fonction régulière. On suppose que (2.1) admet une solution régulière et qu'il peut se réécrire sous la forme

$$
\begin{gathered}
\frac{\partial u}{\partial t}+\frac{\partial}{\partial x}(\mathcal{L} u)+\frac{\partial}{\partial x} \frac{\partial F(u)}{\partial u}=0, \quad x \in \Omega, \quad t>0, \\
u(0, x)=u_{0}(x), \quad x \in \Omega,
\end{gathered}
$$

posée sur $\Omega=\mathbb{R}$ ou bien avec des conditions aux limites périodiques si le domaine est fini, i.e. $\Omega=] 0, \ell[$. On suppose que $\mathcal{L} \in \mathfrak{L}\left(H^{2 m+1}(\Omega), L^{2}(\Omega)\right)$ est un opérateur linéaire auto adjoint qui commute avec $\frac{\partial}{\partial x}$, i.e $\forall v \in H^{2 m+1}(\Omega)$ on a $\frac{\partial}{\partial x} \mathcal{L} v=\mathcal{L} \frac{\partial v}{\partial x}$ pp $x \in \Omega$. Alors

$$
\begin{aligned}
& I_{-1}(u)=\int_{\Omega} u(t, .) d x=\int_{\Omega} u(0, .) d x, \\
& I_{0}(u)=\int_{\Omega} u^{2}(t, .) d x=\int_{\Omega} u^{2}(0, .) d x, \\
& I_{1}(u)=\frac{1}{2}<\mathcal{L} u(t), u(t)>+\int_{\Omega} F(u(t, .)) d x=\frac{1}{2}<\mathcal{L} u(0), u(0)>+\int_{\Omega} F(u(0, .)) d x,
\end{aligned}
$$

où $<., .>$ désigne le produit scalaire dans $L^{2}(\Omega)$.

\section{Preuve. Voir [CCLZ08].}

Par exemple, on observe que les termes linéaires et non-linéaires des équations de KdV (généralisées ou non) satisfont les hypothèses du Lemme 1 ; en particulier on peut réécrire $N(u)=\frac{\partial}{\partial x} \frac{\partial F(u)}{\partial u}$ avec $F(u)=$ $\frac{1}{(p+1)(p+2)} u^{p+2}$. Ce type de résultat s'applique à d'autres équations, telles que celles de Benjamin-Ono ou de Schrödinger, voir par exemple [BK04,DFP81].

Etant donnée une discrétisation de l'intervalle de temps de simulation $[0, T], 0=t_{0}<t_{1}<\cdots<t_{n}<t_{n+1}<$ $\cdots<t_{N}=T$, on note pour tout $n, 0 \leq n \leq N-1, k_{n}=t_{n+1}-t_{n}$ le pas de discrétisation en temps. Dans toute la suite, pour les vecteurs solution discrète on néglige l'indice $j$ du niveau de discrétisation en espace.

\subsection{Schémas de type Crank-Nicolson}

Il existe plusieurs versions de ce schéma d'ordre deux en temps, qui correspondent à une intégration en temps de (2.3) par les méthodes du trapèze et du point milieu.

Schéma de Crank-Nicolson (CN) :

$$
\frac{U^{n+1}-U^{n}}{k_{n}}-\frac{1}{2} L^{(h)}\left(U^{n}+U^{n+1}\right)-\frac{1}{2}\left(N^{(h)}\left(U^{n}\right)+N^{(h)}\left(U^{n+1}\right)\right)=0 .
$$


Schéma de Duràn Sanz-Serna (DSS), [DSS00] :

$$
\frac{U^{n+1}-U^{n}}{k_{n}}-\frac{1}{2} L^{(h)}\left(U^{n}+U^{n+1}\right)-N^{(h)}\left(\frac{U^{n}+U^{n+1}}{2}\right)=0 .
$$

Nous pouvons également considérer un troisième schéma, inspiré de celui proposé dans [DFP81] pour l'équation de Schrödinger.

\section{Schéma de Delfour-Fortin-Payre (DFP) :}

$$
\frac{U^{n+1}-U^{n}}{k_{n}}-\frac{1}{2} L^{(h)}\left(U^{n}+U^{n+1}\right)-\left(F^{(h)}\left(U^{n+1}\right)-F^{(h)}\left(U^{n}\right)\right) \cdot /\left(U^{n+1}-U^{n}\right)=0,
$$

le symbole ./ désignant la division ponctuelle, composantes par composantes et $F^{(h)}$ la discrétisation de l'opérateur $F$. Ce schéma permet de conserver le troisième invariant (énergie), mais pas le deuxième (norme $\left.L^{2}\right)$.

Les schémas (3.23), (3.24) et (3.25) sont inconditionnellement stables mais $U^{n+1}$ est défini comme solution d'un problème non-linéaire. Comme proposé dans Bona et al. [BDKM96,BDKM91,BDKM95,BDK86], on résout ce système à chaque pas de temps par quelques itérations de point fixe; dans la pratique et pour les équations considérées ici, telles que KdV, très peu d'itérations suffisent pour obtenir une bonne approximation du point fixe $U^{n+1}$ (voir Tables 1 et 2 en section 4).

Schéma (CN) et point fixe

\begin{tabular}{|ll|}
\hline Poser & $v_{0}=U^{n}, m=0$ \\
& jusqu'à convergence \\
Calculer & $v_{m+1}=\left(I d-\frac{k_{n}}{2} L^{(h)}\right)^{-1}\left(\left(I d+\frac{k_{n}}{2} L^{(h)}\right) U^{n}+\frac{k_{n}}{2}\left(N^{(h)}\left(U^{n}\right)+N^{(h)}\left(v_{m}\right)\right)\right)$ \\
& $m=m+1$
\end{tabular}

Le schéma de point fixe pour (DSS) s'écrit de façon similaire, en remplaçant $\frac{1}{2}\left(N^{(h)}\left(U^{n}\right)+N^{(h)}\left(v_{m}\right)\right)$ par $N^{(h)}\left(\frac{U^{n}+v_{m}}{2}\right)$.

\section{Schéma de Crank-Nicolson et Splitting}

La décomposition en grandes et petites échelles permet de résoudre itérativement ces problèmes de point fixe en $Y$ et en $Z$ par une méthode de type Gauss-Seidel non-linéaire.

Schéma (CN) et point fixe en $Y$ et en $Z$

\begin{tabular}{|ll|}
\hline Poser & $\left(Y^{n}, Z^{n}\right)^{T}=S^{-1} U^{n}, v_{0}=Y^{n}, w_{0}=Z^{n}, m=0$ \\
Faire & $\left(n l y_{0}, n l z_{0}\right)^{T}=\left(N_{Y}^{(h)}\left(Y^{n}, Z^{n}\right), N_{Z}^{(h)}\left(Y^{n}, Z^{n}\right)\right)^{T}$ \\
Poser & jusqu'à convergence \\
$\left(n l y_{m}, n l z_{m}\right)^{T}=S^{-1} N^{(h)}\left(S \hat{u}_{m}\right)$, avec $\hat{u}_{m}=\left(v_{m}, w_{m}\right)^{T}$ \\
Faire & $v_{m+1}=\left(I d_{1}-\frac{k_{n}}{2} L_{11}^{(h)}\right)^{-1}\left(\left(I d_{1}+\frac{k_{n}}{2} L_{11}^{(h)}\right) Y^{n}+\frac{k_{n}}{2}\left(n l y_{0}+n l y_{m}+L_{12}^{(h)}\left(w_{0}+w_{m}\right)\right)\right)$ \\
Faire & $w_{m+1}=\left(I d_{2}-\frac{k_{n}}{2} L_{22}^{(h)}\right)^{-1}\left(\left(I d_{2}+\frac{k_{n}}{2} L_{22}^{(h)}\right) Z^{n}+\frac{k_{n}}{2}\left(n l z_{0}+n l z_{m}+L_{21}^{(h)}\left(v_{0}+v_{m}\right)\right)\right)$ \\
& $m=m+1$ \\
Poser & $U^{n+1}=S\left(v_{m}, w_{m}\right)^{T}$ \\
\hline
\end{tabular}

En général le schéma (CN) (3.23) ne permet pas de conserver le deuxième invariant (norme $L^{2}$ ) de la solution. En revanche, dans le schéma de (DSS) (3.24), on pourra définir l'opérateur non-linéaire discret $N^{(h)}$ de sorte que les premiers invariants soient conservés au niveau discret. Nous allons décrire comment construire l'opérateur non-linéaire $N^{(h)}$ afin de conserver aussi le deuxième invariant. 
On établit d'abord le résultat suivant :

Lemme 2. Considérons l'équation d'évolution (3.21)-(3.22). Sous les hypothèses du lemme 1, le schéma de Durán Sanz-Serna (DSS) appliqué à l'équation semi-discrétisée en temps

$$
\begin{gathered}
\frac{u^{n+1}-u^{n}}{k_{n}}+\frac{\partial}{\partial x} \mathcal{L}\left(\frac{u^{n+1}+u^{n}}{2}\right)+\frac{\partial}{\partial x} \frac{\partial F}{\partial u}\left(\frac{u^{n+1}+u^{n}}{2}\right)=0, \quad x \in \Omega, \quad n \geq 0 \\
u^{0}=u_{0}(x), \quad x \in \Omega,
\end{gathered}
$$

conserve la masse et la norme $L^{2}$.

Preuve. On pourra consulter [CCLZ08].

La conservation du second et du troisième invariant au niveau discret est liée à la façon dont le terme nonlinéaire est discrétisé. On pose $N^{(h)}(U)=D_{x}^{(h)}\left(D_{U}\left(F^{(h)}(U)\right)\right.$ où $D_{y}$ est une approximation de l'opérateur $\frac{\partial}{\partial y}$ par un schéma aux différences, avec $y=x$ ou $y=U$. En approchant sur la grille $\mathcal{G}_{j}$, pour $k=0, \ldots, 2^{j}-1$, $D_{W}^{(h)}\left(F^{(h)}(W)\right)_{k} \operatorname{par} \frac{\left(F^{(h)}(W)\right)_{k+1}-\left(F^{(h)}(W)\right)_{k-1}}{W_{k+1}-W_{k-1}}$, nous obtenons, dans le cas d'une nonlinéarité en $u^{p} u_{x}$, avec $p \geq 1$ :

$$
D_{W}^{(h)}\left(F^{(h)}(W)\right)_{k}=\frac{1}{(p+1)(p+2)} \frac{W_{k+1}^{p+2}-W_{k-1}^{p+2}}{W_{k+1}-W_{k-1}}=\frac{1}{(p+1)(p+2)} \sum_{j=0}^{p+1} W_{k+1}^{p+1-j} W_{k-1}^{j} .
$$

Un développement de Taylor en $W_{k}$ montre qu'il s'agit d'une formule d'ordre un, voir aussi [CCLZ08].

Lemme 3. On considère pour une grille donnée la discrétisation en espace et en temps de l'équation d'évolution $(3.21)-(3.22)$ :

$$
\begin{array}{r}
\frac{U^{n+1}-U^{n}}{k_{n}}+L^{(h)}\left(\frac{U^{n+1}+U^{n}}{2}\right)+D_{x}^{(h)}\left(D_{U}^{(h)}\left(F^{(h)}\left(U^{n+1}, U^{n}\right)\right)\right)=0 \\
U^{0} \text { donné dans } \mathbb{R}^{2^{j}},
\end{array}
$$

où

- $L^{(h)}$ et $D_{x}^{(h)}$ sont respectivement les matrices (antisymétriques) de discrétisation en espace de $\frac{\partial}{\partial x} \mathcal{L}$ et de $\frac{\partial}{\partial x}$. De plus, $L^{(h)}=D_{x}^{(h)} A^{(h)}$ avec $A^{(h)}$ la matrice symétrique de discrétisation en espace de $\mathcal{L}$.

- $N^{(h)}\left(U^{n+1}, U^{n}\right)=D_{x}^{(h)}\left(D_{U}^{(h)}\left(F^{(h)}\left(U^{n+1}, U^{n}\right)\right)\right)$ est le terme non-linéaire de discrétisation de $\frac{\partial}{\partial x} \frac{\partial F(u)}{\partial u}$ aux points de la grille $\mathcal{G}_{j}$ et au temps $t_{n+1}$.

On suppose de plus que $<D_{x}^{(h)} W, \mathbf{1}>=0, \forall W \in \mathbb{R}^{2^{j}}$ où $\mathbf{1} \in \mathbb{R}^{2^{j}}$ est le vecteur dont toutes les composantes sont égales à 1 et $<., .>$ désigne le produit scalaire dans $\mathbb{R}^{2^{j}}$. Alors le schéma (CN) (3.23) conserve numériquement le premier invariant, celui (DSS) (3.24) les deux premiers et celui (DFP) (3.25) le premier et le troisième.

Preuve. Ces trois schémas conservent le premier invariant par construction, l'équation étant écrite sous forme conservative. En prenant le produit scalaire discret de l'équation (3.24) avec le vecteur $\mathbf{1} \in \mathbb{R}^{2^{j}}$, on obtient pour tout $n \geq 0$

$$
<\frac{U^{n+1}-U^{n}}{k_{n}}, \mathbf{1}>=\sum_{k} U_{k}^{n+1} h_{j}-\sum_{k} U_{k}^{n} h_{j},
$$

$h_{j}$ étant le pas en espace. Par hypothèse sur $D_{x}^{(h)}$, nous avons

$$
<L^{(h)}\left(\frac{U^{n+1}+U^{n}}{2}\right), \mathbf{1}>=<D_{x}^{(h)} A^{(h)}\left(\frac{U^{n+1}+U^{n}}{2}\right), \mathbf{1}>=0,
$$


et aussi

$$
<N^{(h)}(W), \mathbf{1}>=<D_{x}^{(h)}\left(D_{W}^{(h)}\left(F^{(h)}(W)\right)\right), \mathbf{1}>=0, \forall W \in \mathbb{R}^{2^{j}} .
$$

Afin d'obtenir la conservation de la norme $L^{2}$ discrète, on prend le produit scalaire discret de (3.24) avec $\frac{U^{n+1}+U^{n}}{2}$. Cela donne :

$<\frac{U^{n+1}-U^{n}}{k_{n}}, \frac{U^{n+1}+U^{n}}{2}>+<L^{(h)}\left(\frac{U^{n+1}+U^{n}}{2}\right), \frac{U^{n+1}+U^{n}}{2}>+<N^{(h)}\left(\frac{U^{n+1}+U^{n}}{2}\right), \frac{U^{n+1}+U^{n}}{2}>=0$,

avec

$$
<\frac{U^{n+1}-U^{n}}{k_{n}}, \frac{U^{n+1}+U^{n}}{2}>=\frac{\left|U^{n+1}\right|^{2}-\left|U^{n}\right|^{2}}{2 k_{n}}
$$

et, par antisymétrie de $L^{(h)}$,

$$
<L^{(h)}\left(\frac{U^{n+1}+U^{n}}{2}\right), \frac{U^{n+1}+U^{n}}{2}>=0 .
$$

La conservation de la norme $L^{2}$ équivaut à imposer

$$
<N^{(h)}\left(\frac{U^{n+1}+U^{n}}{2}\right), \frac{U^{n+1}+U^{n}}{2}>=0,
$$

c'est-à-dire

$$
<N^{(h)}(W), W>=0, \forall W \in \mathbb{R}^{2^{j}} .
$$

En utilisant (3.28), on a :

$$
\begin{aligned}
<N^{(h)}(W), W> & =<D_{x}^{(h)}\left(D_{W}^{(h)}\left(F^{(h)}(W)\right)\right), W> \\
& =-<D_{W}^{(h)}\left(F^{(h)}(W)\right), D_{x}^{(h)} W> \\
& =-<D_{x}^{(h)} F^{(h)}(W), \mathbf{1}> \\
& =0 .
\end{aligned}
$$

Pour établir la conservation du troisième invariant au niveau discret pour le schéma (DFP) (3.25), on considère la produit scalaire de ce système avec le vecteur $\frac{1}{2} A^{(h)}\left(U^{n+1}+U^{n}\right)+\left(F^{(h)}\left(U^{n+1}\right)-F^{(h)}\left(U^{n}\right)\right) \cdot /\left(U^{n+1}-U^{n}\right)$. Par antysymétrie de $D_{x}^{(h)}$, cela donne :

$$
<\frac{U^{n+1}-U^{n}}{k_{n}}, \frac{1}{2} A^{(h)}\left(U^{n+1}+U^{n}\right)+\left(F^{(h)}\left(U^{n+1}\right)-F^{(h)}\left(U^{n}\right)\right) \cdot /\left(U^{n+1}-U^{n}\right)>=0 .
$$

Or, grâce à la symétrie de $A^{(h)}$,

$$
<\frac{U^{n+1}-U^{n}}{k_{n}}, \frac{1}{2} A^{(h)}\left(U^{n+1}+U^{n}\right)>=\frac{1}{2 k_{n}}\left(<A^{(h)} U^{n+1}, U^{n+1}>-<A^{(h)} U^{n}, U^{n}>\right),
$$

et d'autre part

$$
\begin{aligned}
<\frac{U^{n+1}-U^{n}}{k_{n}},\left(F^{(h)}\left(U^{n+1}\right)-F^{(h)}\left(U^{n}\right)\right) \cdot /\left(U^{n+1}-U^{n}\right)> & =\sum_{k} \frac{U_{k}^{n+1}-U_{k}^{n}}{k_{n}} \frac{\left(F^{(h)}\left(U^{n+1}\right)\right)_{k}-\left(F^{(h)}\left(U^{n}\right)\right)_{k}}{U_{k}^{n+1}-U_{k}^{n}} \\
& =\frac{1}{k_{n}} \sum_{k}\left(F^{(h)}\left(U^{n+1}\right)\right)_{k}-\frac{1}{k_{n}} \sum_{k}\left(F^{(h)}\left(U^{n}\right)\right)_{k} .
\end{aligned}
$$


En sommant ces deux identités et en multipliant par $h_{j}$, on trouve

$$
\frac{1}{2} \sum_{k} L^{(h)} U_{k}^{n+1} U_{k}^{n+1} h_{j}+\sum_{k}\left(F^{(h)}\left(U^{n+1}\right)\right)_{k} h_{j}=\frac{1}{2} \sum_{k} L^{(h)} U_{k}^{n} U_{k}^{n} h_{j}+\sum_{k}\left(F^{(h)}\left(U^{n}\right)\right)_{k} h_{j} .
$$

Ceci ne traduit rien d'autre que la conservation de $\frac{1}{2}<\mathcal{L} u(t), u(t)>+\int_{\Omega} F(u(t,)) d$.$x au niveau discret sur$ chaque intervalle $\left(t_{n}, t_{n+1}\right)$, où l'intégrale en espace est approché par une formule des rectangles.

\subsection{Schémas de type Runge-Kutta}

Nous considérerons les méthodes classiques [CM84, Dem96] de Runge-Kutta à 4 niveaux : RK43, qui est semi-implicite A-stable d'ordre 3, et RK4, qui est explicite A-stable d'ordre 4. Nous évoquons ci-après différentes versions de type splitting de ces schémas et nous présentons en détail deux versions qui généralisent la méthode RK43 dans le cas d'une décomposition de la solution sur deux échelles.

\section{Schéma de splitting RK43-RK4 et de type Driscoll amélioré}

Driscoll propose dans [Dri02] de séparer l'intégration des $Y$ et des $Z$ : son idée consiste à utiliser un schéma RK43 pour l'équation en $Y$ et un schéma RK4 explicite pour l'équation en $Z$. Nous avons testé ce premier schéma proposé par Driscoll mais aussi une variante (plus stable) de celui-ci, qui symétrise le traitement des $Y$ et des $Z$ et qui est présentée ci-dessous.

Le schéma de splitting Driscoll amélioré

\begin{tabular}{|ll|}
\hline Poser & $v_{1}=U^{n},\left(Y^{n}, Z^{n}\right)^{T}=S^{-1} U^{n}, y_{1}=Y^{n}, z_{1}=Z^{n}$ \\
Calculer & $\left(n l y_{1}, n l z_{1}\right)^{T}=S^{-1} N^{(h)}\left(v_{1}\right)$ \\
Calculer & $y_{2}=\left(I d_{1}-\frac{k_{n}}{3} L_{11}^{(h)}\right)^{-1}\left(\left(I d_{1}+\frac{k_{n}}{6} L_{11}^{(h)}\right) y_{1}+\frac{k_{n}}{2}\left(n l y_{1}+L_{12}^{(h)} z_{1}\right)\right)$ \\
Calculer & $z_{2}=\left(I d_{2}-\frac{k_{n}}{3} L_{22}^{(h)}\right)^{-1}\left(\left(I d_{2}+\frac{k_{n}}{6} L_{22}^{(h)}\right) z_{1}+\frac{k_{n}}{2}\left(n l z_{1}+L_{21}^{(h)} y_{2}\right)\right)$ \\
Poser & $v_{2}=S\left(y_{2}, z_{2}\right)^{T}$ \\
Calculer & $\left(n l y_{2}, n l z_{2}\right)^{T}=S^{-1} N^{(h)}\left(v_{2}\right)$ \\
Calculer & $y_{3}=\left(I d_{1}-k_{n} L_{11}^{(h)}\right)^{-1}\left(\left(I d_{1}+\frac{k_{n}}{2} L_{11}^{(h)}\right) y_{1}-k_{n} L_{11}^{(h)} y_{2}+\frac{k_{n}}{2}\left(n l y_{2}+L_{12}^{(h)} z_{2}\right)\right)$ \\
Calculer & $z_{3}=\left(I d_{2}-k_{n} L_{22}^{(h)}\right)^{-1}\left(\left(I d_{2}+\frac{k_{n}}{2} L_{22}^{(h)}\right) z_{1}-k_{n} L_{22}^{(h)} z_{2}+\frac{k_{n}}{2}\left(n l z_{2}+L_{21}^{(h)} y_{3}\right)\right)$ \\
Poser & $v_{3}=S\left(y_{3}, z_{3}\right)^{T}$ \\
Calculer & $\left(n l y_{3}, n l z_{3}\right)^{T}=S^{-1} N^{(h)}\left(v_{3}\right)$ \\
Calculer & $y_{4}=\left(I d_{1}-\frac{k_{n}}{3} L_{11}^{(h)}\right)^{-1}\left(y_{1}+\frac{2 k_{n}}{3} L_{11}^{(h)} y_{3}+k_{n}\left(n l y_{3}+L_{12}^{(h)} z_{3}\right)\right)$ \\
Calculer & $z_{4}=\left(I d_{2}-\frac{k_{n}}{3} L_{22}^{(h)}\right)^{-1}\left(z_{1}+\frac{2 k_{n}}{3} L_{22}^{(h)} z_{3}+k_{n}\left(n l z_{3}+L_{21}^{(h)} y_{4}\right)\right)$ \\
Poser & $v_{4}=S\left(y_{4}, z_{4}\right)^{T}$ \\
Calculer & $\left(n l y_{4}, n l z_{4}\right)^{T}=S^{-1} N^{(h)}\left(v_{4}\right)$ \\
Définir & $Y^{n+1}=Y^{n}+\frac{k_{n}}{6}\left(n l y_{1}+n l y_{4}+L_{11}^{(h)}\left(y_{1}+y_{4}\right)+L_{12}^{(h)}\left(z_{1}+z_{4}\right)\right)$ \\
& $\quad+\frac{k_{n}}{3}\left(n l y_{2}+n l y_{3}+L_{11}^{(h)}\left(y_{2}+y_{3}\right)+L_{12}^{(h)}\left(z_{2}+z_{3}\right)\right)$ \\
Définir & $Z^{n+1}=Z^{n}+\frac{k_{n}}{6}\left(n l z_{1}+n l z_{4}+L_{21}^{(h)}\left(y_{1}+y_{4}\right)+L_{22}^{(h)}\left(z_{1}+z_{4}\right)\right)$ \\
Poser & $\quad U^{n+1}=\frac{k_{n}}{3}\left(n l z_{2}+n l z_{3}+L_{21}^{(h)}\left(y_{2}+y_{3}\right)+L_{22}^{(h)}\left(z_{2}+z_{3}\right)\right)$ \\
& \\
&
\end{tabular}




\section{Schémas RK43 avec seuillage et de type "multiniveaux GNL"}

Enfin, nous cherchons à écrire des algorithmes qui effectuent un traitement particulier des $Z$. Un premier algorithme, qui s'inspire des analyses multi-résolutions en traitement d'image, consiste à négliger les détails $Z$ inférieurs à un seuil $\varepsilon$ donné et à appliquer un schéma RK43 sur la partie de la solution relative à $Y$ et aux détails $Z$ supérieurs au seuil. Le schéma classique RK43 sera donc appliqué à un système d'équations généralement de taille inférieure au système d'origine (si le seuillage est bien choisi). Un dernier algorithme s'inspire des méthodes multi-niveaux de type Galerkin non-linéaire (GNL). Il s'agit de décomposer la solution en deux parties, comme dans le cas précédent, mais cette fois-ci, sous le critère local de petitesse des $Z$, on résout par le schéma RK43 seulement les équations relatives aux $Y$ complétées par les détails $Z$ supérieurs au seuil donné. En revanche, les composantes $Z$ inférieures au seuil seront figées sur l'intervalle $\left(t_{n}, t_{n+1}\right)$ (c'est-à-dire $Z_{k}^{n+1}=Z_{k}^{n}$ pour les composantes $k$ telle que $\left|Z_{k}^{n}\right|<\varepsilon$ ). Ici, le terme non-linéaire est différent par rapport au cas précédent avec seuillage, car maintenant les $Z$ sont figées et pas annulées. On remarque enfin la présence du terme linéaire $L_{12}^{(h)} Z^{n}$ qui intervient comme un terme source, en explicite. L'analyse de la solution nodale étant faite à chaque pas de temps, sa décomposition en $Y$ et $Z$ varie au cours du temps, ainsi que les composantes figées. Cela permet donc de faire avancer globalement toute la solution.

Le schéma RK43 type "multiniveaux GNL"

\begin{tabular}{|ll|}
\hline Poser & $v_{1}=U^{n},\left(Y^{n}, Z^{n}\right)^{T}=S^{-1} U^{n}, y_{1}=Y^{n}$ \\
Calculer & $\left(n l y_{1}, n l z_{1}\right)^{T}=S^{-1} N^{(h)}\left(v_{1}\right)$ \\
Calculer & $y_{2}=\left(I d_{1}-\frac{k_{n}}{3} L_{11}^{(h)}\right)^{-1}\left(\left(I d_{1}+\frac{k_{n}}{6} L_{11}^{(h)}\right) y_{1}+\frac{k_{n}}{2}\left(n l y_{1}+L_{12}^{(h)} Z^{n}\right)\right)$ \\
Poser & $v_{2}=S\left(y_{2}, Z^{n}\right)^{T}$ \\
Calculer & $\left(n l y_{2}, n l z_{2}\right)^{T}=S^{-1} N^{(h)}\left(v_{2}\right)$ \\
Calculer & $y_{3}=\left(I d_{1}-k_{n} L_{11}^{(h)}\right)^{-1}\left(\left(I d_{1}+\frac{k_{n}}{2} L_{11}^{(h)}\right) y_{1}-k_{n} L_{11}^{(h)} y_{2}+\frac{k_{n}}{2}\left(n l y_{2}+L_{12}^{(h)} Z^{n}\right)\right)$ \\
Poser & $v_{3}=S\left(y_{3}, Z^{n}\right)^{T}$ \\
Calculer & $\left(n l y_{3}, n l z_{3}\right)^{T}=S^{-1} N^{(h)}\left(v_{3}\right)$ \\
Calculer & $y_{4}=\left(I d_{1}-\frac{k_{n}}{3} L_{11}^{(h)}\right)^{-1}\left(y_{1}+\frac{2 k_{n}}{3} L_{11}^{(h)} y_{3}+k_{n}\left(n l y_{3}+L_{12}^{(h)} Z^{n}\right)\right)$ \\
Poser & $v_{4}=S\left(y_{4}, Z^{n}\right)^{T}$ \\
Calculer & $\left(n l y_{4}, n l z_{4}\right)^{T}=S^{-1} N^{(h)}\left(v_{4}\right)$ \\
Définir & $Y^{n+1}=Y^{n}+\frac{k_{n}}{6}\left(n l y_{1}+n l y_{4}+L_{11}^{(h)}\left(y_{1}+y_{4}\right)\right)$ \\
& $\quad+\frac{k_{n}}{3}\left(n l y_{2}+n l y_{3}+L_{11}^{(h)}\left(y_{2}+y_{3}\right)\right)+k_{n} L_{12}^{(h)} Z^{n}$ \\
Poser & $U^{n+1}=S\left(Y^{n+1}, Z^{n}\right)^{T}$ \\
\hline
\end{tabular}

\section{Applications}

Nous considérons ici deux types d'équations, l'une dispersive (KdV), l'autre dissipative (KS). Dans les deux cas, le but de l'étude est de comparer la stabilité numérique des schémas multiniveaux proposés en section 3.2 avec celle des schémas classiques de la section 3.1. Des schémas d'Euler semi-implicites peuvent aussi être considérés. 


\subsection{L'équation de Korteweg de Vries (KdV)}

L'équation de KdV généralisée, écrite sous forme conservative est :

$$
\left\{\begin{aligned}
u_{t}+\partial_{x}\left(\mu u_{x x}+\frac{1}{p+1} u^{p+1}\right) & =0 & & x \in \mathbb{R}, t>0 \\
u(0, x) & =u_{0}(x) & & x \in \mathbb{R}
\end{aligned}\right.
$$

avec $\mu>0$ et $p \geq 1$. Comme dans [BDKM95], on prend comme donnée initiale le soliton

$$
u_{0}(x)=A \operatorname{sech}^{2 / p}\left(K\left(x-x^{0}\right)\right)
$$

alors la solution exacte de (4.31) est :

$$
u(t, x)=A \operatorname{sech}^{2 / p}\left(K\left(x-x^{0}-C t\right)\right), x \in \mathbb{R}, t>0
$$

avec la vitesse $C$ de propagation du soliton :

$$
C=\frac{2 A^{p}}{(p+1)(p+2)} \text { et } K=\frac{p}{2} \sqrt{\frac{C}{\mu}} .
$$

Afin de simuler la solution physique qui est posée sur tout $\mathbb{R}$, on considère un domaine $\Omega=] 0, \ell[$ avec $\ell$ assez grand afin que le soliton reste "complètement" à l'intérieur du domaine même pour des simulations en temps très longues. Les conditions aux limites sont prises périodiques, mais des conditions de Dirichlet homogènes peuvent également être imposées. La solution initiale est un soliton centré en $x^{0}=\ell / 2$. On considérera les valeurs $\mu=10^{-4}, p=4$ et l'amplitude $A=0.8$; pour cette valeur de $A$, la solution est définie pour tout temps. Dans les simulations suivantes, on prendra $\ell=4$ ou $\ell=8$ en fonction du temps d'arrêt $T$.

Pour des solutions assez régulières, les invariants de (4.31) s'écrivent comme dans le lemme 1. En particulier, le troisième invariant devient

$$
I_{1}(u)=\int_{0}^{\ell}\left(u^{p+2}-\frac{(p+1)(p+2) \mu}{2} u_{x}^{2}\right) d x .
$$

Sur la grille $\mathcal{G}_{j}$, la version discrète du troisième invariant s'écrit :

$$
I_{1}\left(U_{j}^{n}\right)=h_{j} \sum_{k=0}^{2^{j}-1}\left(\left(U_{j, k}^{n}\right)^{p+2}-\frac{(p+1)(p+2) \mu}{2} \frac{\left(U_{j, k+1}^{n}-U_{j, k-1}^{n}\right)^{2}}{4 h_{j}^{2}}\right)
$$

avec $h_{j}$ le pas de discrétisation en espace.

Dans un premier temps, nous testons les schémas classiques (CN), (DSS) et (RK43) sans séparation des échelles. Le but est de comparer leur stabilité sur des très long intervalles de temps, en analysant l'erreur commise sur les invariants. Ensuite, nous appliquons les différentes variantes multiniveaux proposées en section 3, en tenant compte du découpage entre grandes et petites échelles. Globalement, on peut conclure que les schémas classiques (CN), (DSS) et (RK43) permettent de bien simuler la solution de (4.31) sur des longs intervalles de temps lorsque le pas d'espace $h_{j}$ et l'ordre de discrétisation des opérateurs sont choisis convenablement. Dans toutes les simulations faites, on prend une discrétisation des opérateurs d'ordre quatre sauf pour le schéma (DSS), pour lequel nous avons montré qu'il conserve exactement le second invariant $I_{0}$ si les opérateurs sont discrétisés à l'ordre deux. Dans les simulations présentées le pas de temps est fixe, c'est-à-dire $k_{n}=\delta t, \forall n \geq 0$.

\subsubsection{Schéma de Crank-Nicolson (CN)}

On a vu en section 3.1 que le schéma $(\mathrm{CN})$ conserve seulement le premier invariant. Afin de contrôler la conservation numérique des invariants $I_{0}$ et $I_{1}$, on regarde l'évolution de l'erreur relative commise sur ces deux 
invariants :

$$
E_{0}(t)=\frac{\left|I_{0}(u(t))-I_{0}(u(0))\right|}{\left|I_{0}(u(0))\right|}, \quad E_{1}(t)=\frac{\left|I_{1}(u(t))-I_{1}(u(0))\right|}{\left|I_{1}(u(0))\right|} .
$$

Dans la Table 1 on donne les erreurs relatives au temps $t=15 E_{0}(t=15)$ et $E_{1}(t=15)$, en comparant la précision de l'algorithme de Crank-Nicolson $(\mathrm{CN})$ pour des discrétisations spatiales des opérateurs d'ordre deux et quatre. La boucle relative aux itérations de Picard est réalisée avec un nombre d'itérations fixe, donné au départ et noté $k \max$. L'initialisation du point fixe est donnée par la formule d'extrapolation d'ordre deux $v_{0}=\left(3 U^{n}-U^{n-1}\right) / 2$, sauf pour la première itération en temps pour laquelle on choisit $v_{0}=U^{0}$ et on réalise $k \max +2$ itérations internes. Ici, le pas spatial est $h_{j}=2^{-8}$ et celui temporel $\delta t=0,002$.

\begin{tabular}{|c|c|c|c|}
\hline & $k \max =3$ & $k \max =4$ & $k \max =10$ \\
\hline ORDRE 4 & \multicolumn{3}{|l|}{} \\
\hline$E_{0}(t=15)$ & $7 \cdot 10^{-7}$ & $8 \cdot 10^{-9}$ & $4 \cdot 10^{-10}$ \\
\hline$E_{1}(t=15)$ & 0,1158 & $3 \cdot 10^{-4}$ & $9 \cdot 10^{-4}$ \\
\hline \hline ORDRE 2 & \multicolumn{3}{|l}{} \\
\hline$E_{0}(t=15)$ & $4 \cdot 10^{-6}$ & $3 \cdot 10^{-6}$ & $3 \cdot 10^{-6}$ \\
\hline$E_{1}(t=15)$ & 0,0316 & 0,0324 & 0,0324 \\
\hline
\end{tabular}

TABLE 1. Erreurs relatives $E_{0}(t=15)$ et $E_{1}(t=15)$, schéma $(\mathrm{CN})$.

On constate que la conservation numérique du deuxième et troisième invariants est satisfaisante seulement à l'ordre quatre et pour $k \max \geq 4$. La précision numérique sur deuxième invariant s'obtient facilement, en revanche il est important de contrôler le troisième invariant pour s'assurer de la stabilité des calculs sur des longs intervalles de temps.

\subsubsection{Schéma de Duràn Sanz-Serna (DSS)}

Le lemme 3 montre que la conservation formelle du deuxième invariant n'est possible que si les opérateurs sont discrétisés à l'ordre deux. Fixés comme auparavant $\delta t=0,002$ et $h_{j}=2^{-8}$, la partie supérieure de la Table 2 donne les erreurs relatives $E_{0}(t=15)$ et $E_{1}(t=15)$ en fonction du nombre d'itérations internes $k m a x$, tandis que dans la partie inférieure de la Table 2 on fixe $k \max =4$, et on détermine les erreurs relatives en fonction d'un pas d'espace $h_{j}$ variable. A cause de l'ordre de discrétisation des opérateurs, afin d'obtenir des résultats satisfaisants, le schéma requiert un pas d'espace très petit et un nombre suffisant d'itérations internes afin que l'erreur $E_{0}$ sur le second invariant soit de l'ordre de l'erreur machine.

\begin{tabular}{|c|c|c|c|}
\hline$h_{j}=2^{-8}$ & $k \max =3$ & $k \max =4$ & $k \max =10$ \\
\hline$\overline{E_{0}(t=15)}$ & $3 \cdot 10^{-7}$ & $2 \cdot 10^{-9}$ & $10^{-13}$ \\
\hline$E_{1}(t=15)$ & 0,91 & 0,91 & 0,91 \\
\hline$k \max =4$ & $h_{j}=2^{-8}$ & $h_{j}=2^{-9}$ & $h_{j}=2^{-10}$ \\
\hline$\overline{E_{0}(t=15)}$ & $2 \cdot 10^{-9}$ & $5 \cdot 10^{-9}$ & $7 \cdot 10^{-9}$ \\
\hline$\overline{E_{1}(t=15)}$ & 0,91 & 0,29 & 0,0796 \\
\hline
\end{tabular}

TABle 2. Erreurs relatives $E_{0}(t=15)$ et $E_{1}(t=15)$, schéma (DSS).

\subsubsection{Schéma classique de Runge-Kutta (RK43)}

Le schéma (RK43) ne conserve formellement que le premier invariant. Afin de contrôler la conservation numérique des invariants $I_{0}$ et $I_{1}$, on donne dans la Table 3 les erreurs relatives $E_{0}(t=15)$ et $E_{1}(t=15)$, en les comparant sur différentes grilles de discrétisation, avec $\delta t=0,001$ fixé et des opérateurs discrétisés à l'ordre 
quatre. Au cours des simulations effectuées, on observe que la conservation numérique du deuxième invariant est assez satisfaisante, en revanche celle du troisième invariant se dégrade par rapport au schéma $(\mathrm{CN})$. De plus, elle se dégrade aussi si on fixe $\delta t$ et on diminue seulement le $h_{j}$ (comme le montre la dernière valeur dans la Table ). En effet, afin de réduire l'erreur relative $E_{1}$, il faut raffiner en même temps le pas d'espace et le pas de temps.

\begin{tabular}{|c|c|c|c|}
\hline & $h_{j}=2^{-7}$ & $h_{j}=2^{-8}$ & $h_{j}=2^{-9}$ \\
\hline$E_{0}(t=15)$ & $10^{-7}$ & $10^{-8}$ & $10^{-8}$ \\
\hline$E_{1}(t=15)$ & 0,0151 & 0,0013 & 0,0364 \\
\hline
\end{tabular}

TABLE 3. Erreurs relatives $E_{0}(t=15)$ et $E_{1}(t=15)$, schéma (RK43).

\subsubsection{Plusieurs schémas en temps avec séparation des échelles}

Il s'agit ici d'une première analyse des schémas multiniveaux sur l'équation de KdV, c'est la raison pour laquelle on se limite à des tests numériques qui ne considèrent qu'une décomposition sur deux grilles. La généralisation à plusieurs grilles est possible, mais elle ne sera justifiée que si les schémas multiniveaux sont déjà stables et précis pour seulement deux grilles. La stabilité et la précision concernent toujours la conservation des trois premiers invariants. Dans cette section, nous présentons les tests effectués sur plusieurs algorithmes en temps associés à une décomposition de la solution selon les relations (2.16).

\section{Schéma de Crank-Nicolson en Y-Z}

La décomposition en grandes et petites échelles permet de résoudre itérativement les problèmes de point fixe en $Y$ et en $Z$ (voir le schéma $(\mathrm{CN})$ et point fixe en $Y$ et en $Z$ en section 3.1). Néanmoins, ce schéma est beaucoup moins stable que celui classique (noté $(\mathrm{CN})$ ). Par exemple, pour $h_{j}=2^{-8}$ et $k$ max $=4$ fixés, l'algorithme ne converge qu'en considérant un pas de temps très petit $\left(\delta t \leq 2 \cdot 10^{-4}\right)$; en revanche, il diverge si $\delta t=3 \cdot 10^{-4}$. Le problème pratique réside en la mise au point d'algorithmes de point fixe efficaces en $Y$ et en $Z$.

\section{Schéma de Runge-Kutta (RK43) et seuillage}

On considère, à chaque itération en temps, la solution nodale $U_{j+1}^{n}$ décomposée selon $Y_{j}^{n}$ et $Z_{j}^{n}$. Les vecteurs $Y_{j}^{n}$ et $Z_{j}^{n}$ de $\mathbb{R}^{2^{j}}$ contiennent soit les coefficients ondelettes relatifs aux relations (2.15), soit les inconnues incrémentales données par les relations (2.16). On applique un critère de seuillage sur les coefficients ondelettes de type :

$$
\text { on pose } Z_{j, k}^{n}=0 \quad \text { si } \quad\left|Z_{j, k+l}^{n}\right|<\varepsilon \quad \text { pour } l=-1,0,+1 .
$$

La précision $\varepsilon$ du seuillage est fixée au départ et dépend du niveau de discrétisation du problème. Pour ce qui concerne la technique de seuillage, on n'élimine pas un coefficient ondelettes, même s'il est inférieur au seuil fixé, lorsqu'il est voisin immédiat d'un autre coefficient de valeur supérieure au seuil. En choisissant $|l|>1$, on garderait plusieurs voisins et pas seulement deux, comme actuellement avec $|l| \leq 1$. Par le critère (4.32), on réussit à éliminer plus de $90 \%$ des coefficients $Z_{j, k}^{n}$. Dans la Figure 2 , on peut voir que les coefficients $Z_{j, k}^{n}$ conservés (représentés par des croix) sont localisés autour du soliton. Cela demeure vrai au cours du temps.

Sans le critère qui prend en compte les voisins immédiats des coefficients sous le seuil $\varepsilon$, la solution calculée n'est plus correcte. On remarque cela dans la partie gauche de la Figure 3 : dans le graphique de la solution calculée on observe une vitesse de propagation et une amplitude inférieures à celles de la solution exacte à $t=50$. Le même constat peut être fait sur l'évolution de l'erreur $\left\|u-u_{e x}\right\|_{L^{2}}^{2}$ et sur l'évolution de l'erreur relative $E_{1}(t)$ du troisième invariant (partie droite de la Figure 3 ).

En éliminant, grâce à (4.32), des coefficients ondelettes, on se ramène à résoudre, à chaque itération en temps et pour chaque étape du schéma RK43, un système linéaire de taille inférieure à celle du problème sur la grille 

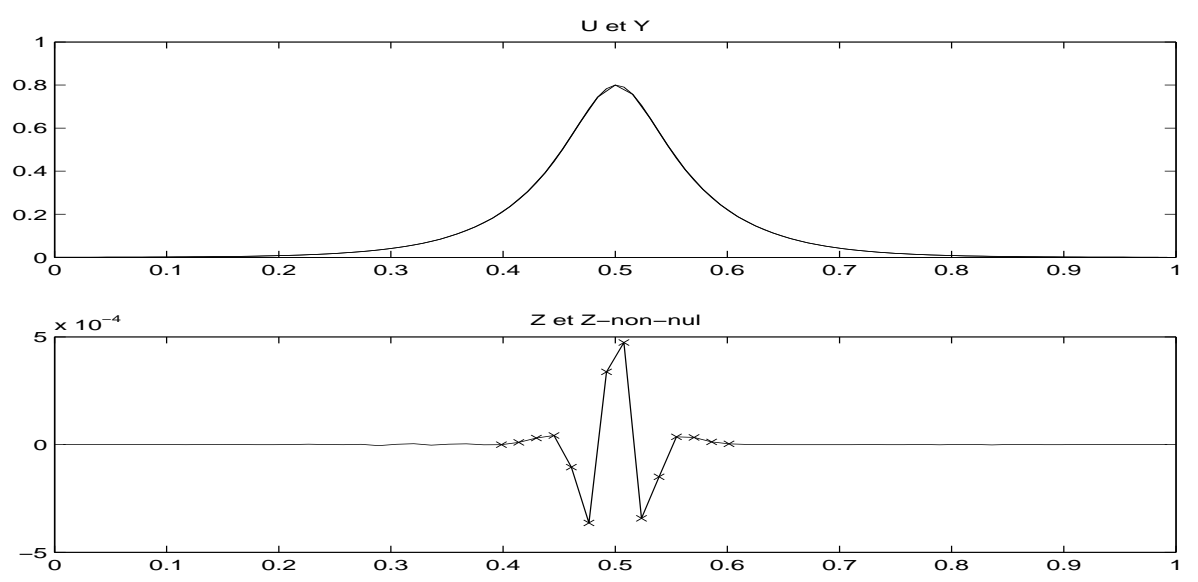

Figure 2. En haut: représentation de la solution $U^{n}(x)$ et $Y^{n}(x)$; en bas : représentation de $Z^{n}(x)$ et des coefficients conservés (croix).
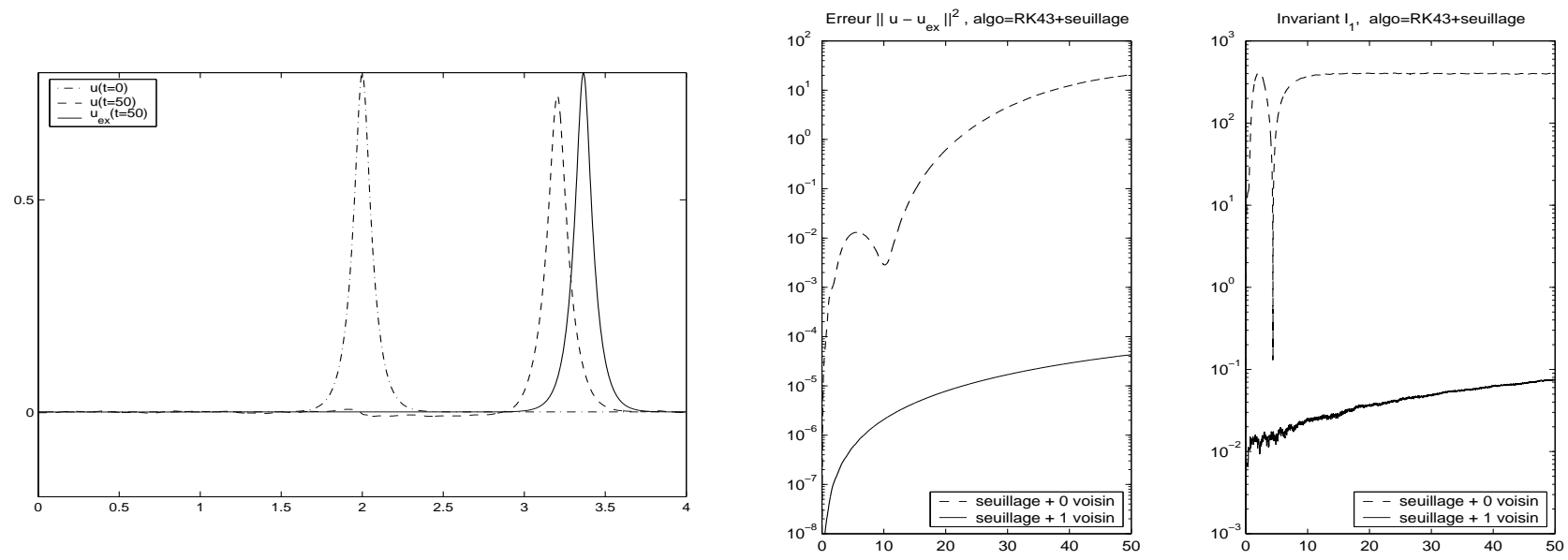

Figure 3. A gauche : Solution calculée et solution exacte à $t=50$. A droite : évolution de l'erreur sur la solution $\left\|u-u_{e x}\right\|_{L^{2}}^{2}$ et de l'erreur relative $E_{1}(t)$ si l'on ajoute aucun ou un voisin au critère de seuillage.

fine de niveau $j+1$. Cette taille correspond à celle du vecteur $Y_{j}^{n}$ augmenté du nombre des coefficients $Z_{j, k}^{n}$ de valeur absolue supérieure au seuil. Néanmoins, le coût relatif à l'assemblage du terme non-linéaire reste invarié car il doit toujours être effectué sur la base nodale, à partir de la solution reconstruite sur la grille fine après l'étape de seuillage.

On fixe $\ell=8, h_{j}=2^{-8}, \delta t=0,002$ et $\varepsilon=10^{-7}$. On compare d'abord l'influence de l'ordre d'interpolation choisie pour effectuer la décomposition de la solution $U_{j+1}^{n}$ en $Y_{j}^{n}$ et $Z_{j}^{n}$. On trace en Figure 4 l'évolution de l'erreur $\left\|u-u_{e x}\right\|_{L^{2}}^{2}$ et de l'erreur relative $E_{1}(t)$ sur le troisième invariant pour des interpolations d'ordre 4 et 6. On remarque que, pour avoir un calcul stable sur des longs intervalles de temps, il faut considérer des interpolations d'ordre supérieur à celui de la discrétisation des opérateurs (ordre 4 dans ce cas). On compare ensuite l'influence du paramètre de seuillage $\varepsilon$. En Figure 5 on représente l'évolution de l'erreur relative sur le troisième invariant $E_{1}(t)$, en considérant différentes valeurs de $\varepsilon$. Si on élimine les coefficients $Z_{j, k}^{n}$ en utilisant des valeurs de $\varepsilon>10^{-6}$, le schéma n'est pas stable, car on néglige des détails de la solution qui contiennent 
encore beaucoup d'énergie. Pour $10^{-6} \leq \varepsilon<10^{-7}$, l'erreur relative $\left\|u-u_{e x}\right\|_{L^{2}}^{2}$ reste bornée par $10^{-2}$, mais soit $E_{1}(t)$ devient trop grand, soit il oscille beaucoup. Dans ce cas, l'invariant $I_{1}(t)$ n'est pas bien conservé, même si sa valeur oscille autour de la valeur initiale $I_{1}(t=0)$. Des valeurs de $\varepsilon$ entre $10^{-8}$ et $10^{-7}$ sont appropriées pour bien capturer le soliton sur des longs intervalles de temps.
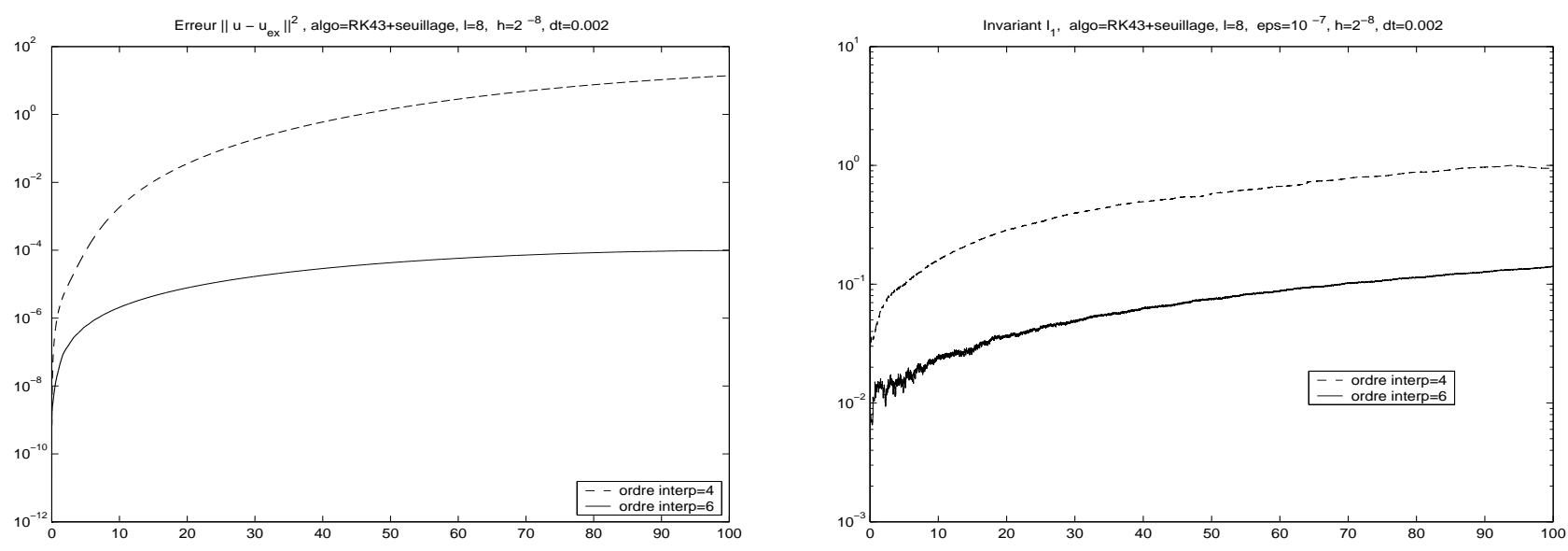

FiguRE 4. A gauche, évolution de l'erreur sur la solution $\left\|u-u_{e x}\right\|_{L^{2}}^{2}$; à droite, évolution de l'erreur relative $E_{1}(t)$ pour des interpolations d'ordre 4 et 6 .

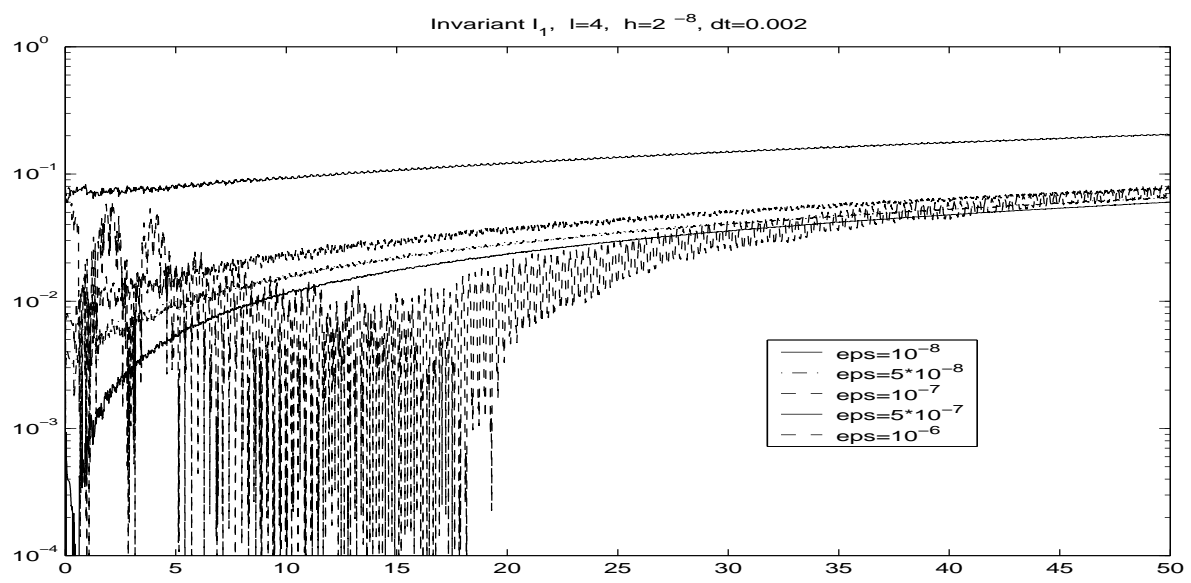

Figure 5. Evolution en temps de l'erreur du troisième invariant $E_{1}(t)$ pour différentes valeurs du paramètre de seuillage $\varepsilon$.

En conclusion, vu les résultats positifs de ce schéma avec seuillage, dans un travail futur nous pourrons utiliser cette approche pour réaliser facilement du raffinement de maillage, ce qui constitue un développement nécessaire pour le calcul de solutions très localisées en espace.

\section{Schémas de splitting "Driscoll et Driscoll amélioré" avec seuillage}

Pour cette classe de schémas, le critère de seuillage permet des séparer en deux parties la solution : les grandes échelles de la solution sont les composantes de $Y_{j}^{n}$ et les $Z_{j, k}^{n}$ de valeur absolue supérieure au seuillage $\varepsilon$, tandis 
que les $Z_{j, k}^{n}$ de valeur absolue inférieure au seuillage sont associées aux petites échelles. Ces dernières sont calculées dans des schémas qui s’inspirent des travaux de Driscoll [Dri02], tandis qu'elles étaient éliminées dans l'algorithme (RK43) avec seuillage.

A partir de cette décomposition des inconnues, on résout à chaque étape d'un schéma de type (RK43) deux systèmes non-linéaires couplés. Le système d'équations correspondant aux coefficients $\left|Z_{j, k}^{n}\right|<\varepsilon$ sera résolu soit par l'algorithme proposé dans [Dri02] (un schéma explicite RK4 pour la mise à jour des petites échelles complète le schéma RK43 pour résoudre les grandes échelles), soit par l'algorithme de splitting, nommé Driscoll amélioré, proposé en section 3.2. La stabilité des ces deux schémas est comparable, mais bien inférieure à celle des algorithmes classiques ou de l'algorithme (RK43) avec seuillage. La Table 4 résume la stabilité de ces différents algorithmes. On remarque que pour $h_{j}=2^{-8}$ et $\epsilon=10^{-7}$, les pas de temps pour assurer la stabilité sont beaucoup plus petits que ceux utilisés pour les schémas (RK43) classique ou (RK43) avec seuillage. Cette approche n'est donc pas adéquate pour la simulation d'équations dispersives, telle que KdV.

\begin{tabular}{|c|c|c|c|}
\hline algo & $\delta t=0,001$ & $\delta t=0,0004$ & $\delta t=0,0003$ \\
\hline Driscoll & NAN & NAN & stable \\
\hline Driscoll + seuillage & Z oscillent trop & $\left|Z_{j, k}^{n}\right|>\varepsilon$ pour tout $k$ & stable \\
\hline Driscoll amélioré & NAN & NAN & stable \\
\hline Driscoll amélioré + seuillage & Z oscillent trop & $\left|Z_{j, k}^{n}\right|>\varepsilon$ pour tout $k$ & stable \\
\hline
\end{tabular}

TABLE 4. Stabilité des schémas de type Driscoll en fonction du pas $\delta t$.

\section{Schéma RK43 type "multiniveaux GNL"}

Ce schéma est un algorithme multiniveaux de type Galerkin non-linéaire (GNL) [CLT97]. Il s'agit de décomposer la solution en deux parties, comme dans le cas précédent. Cette fois-ci, on résout à chaque étape du schéma (RK43) un seul système non-linéaire, celui relatif à $Y_{j}^{n}$ et aux coefficients $Z_{j, k}^{n}$ supérieurs au seuil, pendant que les $Z_{j, k}^{n}$ de valeur absolue inférieure au seuil sont figés sur l'intervalle $\left(t_{n}, t_{n+1}\right)$ (c'est-à-dire $Z_{j, k}^{n+1}=Z_{j, k}^{n}$ pour les composantes $k$ telles que $\left|Z_{j, k}^{n}\right|<\varepsilon$ ). Puisque l'analyse de la solution $U_{j+1}^{n}$ est faite à chaque pas de temps, sa décomposition en grandes et petites échelles varie au cours du temps. Ce schéma de type GNL met à jour les différentes composantes non figées de la solution, mais puisque les composantes figées varient au cours du temps, cela permet de faire avancer tous les modes de la solution.

Cet algorithme présente une stabilité comparable à celle de l'algorithme (RK43) avec seuillage (ici $\delta t=0,002)$. Néanmoins, sont utilisation n'est pas envisageable car ce schéma ne conserve pas correctement le troisième invariant, comme on peut l'observer dans la Figure 6.

\subsection{L'équation de Kuramoto-Sivashinski (KS)}

Nous considérons ici une équation dissipative pour montrer que la présence d'un terme d'amortissement régularisant permet de mettre en œuvre des schémas simples de type GNL, ce qui n'est pas le cas avec des équations dispersives telles que KdV, comme vu plus haut, mais aussi Benjamin-Ono ou Schrödinger (voir aussi [CCLZ08]).

L'équation de Kuramoto-Sivashinsky décrit la propagation d'un front de flamme d'une combustion, sous certaines hypothèses. En domaine périodique elle s'écrit sous la forme

$$
\begin{aligned}
u_{t}+u_{x x x x}+u_{x x}+\frac{1}{2}\left(u_{x}\right)^{2}=0, & x \in] 0, \ell[, \quad t>0, \\
u(0, x)=u_{0}(x), & x \in] 0, \ell[, \\
\frac{\partial^{j} u}{\partial x^{j}}(t, x+\ell)=\frac{\partial^{j} u}{\partial x^{j}}(t, x), & j=0, \ldots, 3 .
\end{aligned}
$$



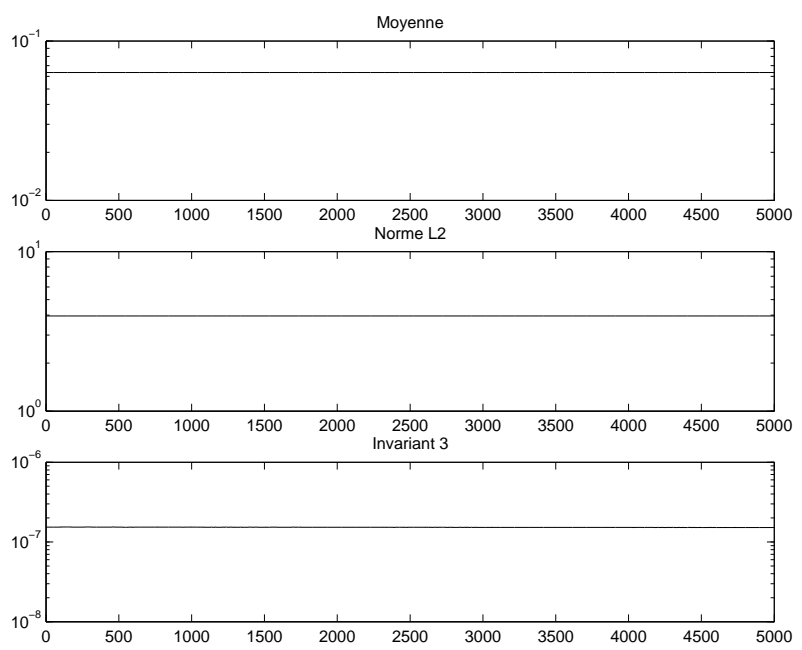
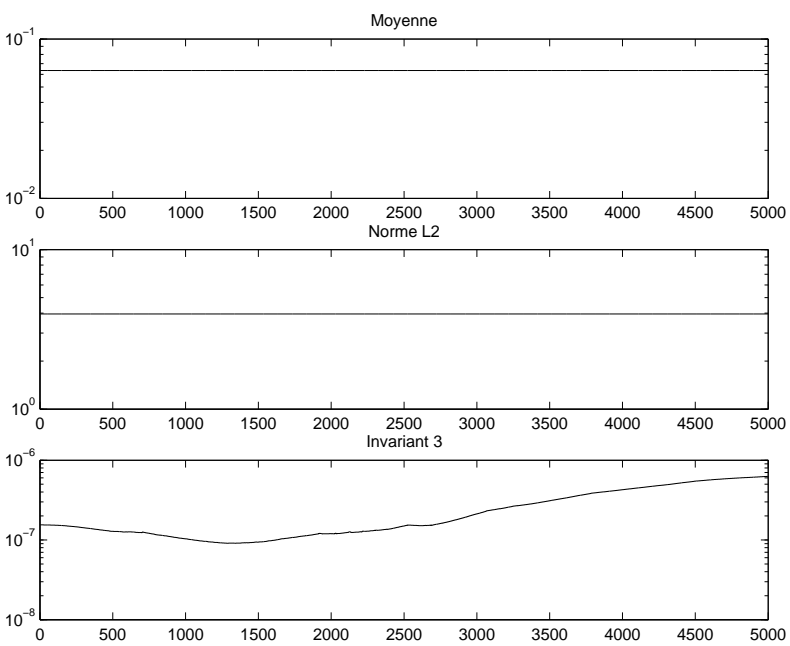

Figure 6. A gauche : évolution en temps des trois invariants de KDV pour l'algorithme RK43 avec seuillage. A droite : évolution en temps des trois invariants de KDV pour l'algorithme RK43 type "multiniveaux GNL". En abscisse, le nombre d'itérations en temps, avec $\delta t=0,002$.

Cette équation comporte un terme dissipatif : elle a été étudiée dans le cadre de la théorie des variétées inertielles [Tem97] mais aussi, d'un point de vue numérique, dans l'optique de la mise en œuvre de méthodes de type Galerkin non-linéaire, voir par exemple [JRT01].

\subsubsection{Discrétisation du problème}

Nous décrivons ici les schémas obtenus par discrétisation spatiale en différences finies et nous effectuons une décomposition en échelles à l'aide d'inconnues incrémentales (II ou IU), telles que décrites dans la section 2.2.2. Pour valider nos résultats nous avons également développé un code en Fourier, qui donne des résultats numériques comparables.

On note $L^{(h)}$ la matrice de discrétisation de l'opérateur $\partial_{x x x x}+\partial_{x x}$ à différents ordres sur la grille fine, dans la base nodale. Pour la discrétisation en temps du système différentiel (2.3), nous utiliserons les deux schémas suivants, avec ou sans séparation des échelles :

Schéma d'Euler semi-implicite en $U$

Calculer $\left.\quad U^{n+1}=\left(I d-k_{n} L^{(h)}\right)^{-1}\left(U^{n}+k_{n} N^{(h)}\left(U^{n}\right)\right)\right)$

Schéma d'Euler semi-implicite en $Y$ et en $Z$

\begin{tabular}{|ll|}
\hline Poser & $\left(Y^{n}, Z^{n}\right)^{T}=S^{-1} U^{n}$ \\
Faire & $(n l y, n l z)^{T}=S^{-1} N^{(h)}\left(U^{n}\right)$ \\
Calculer & $Y^{n+1}=\left(I d_{1}-k_{n} L_{11}^{(h)}\right)^{-1}\left(Y^{n}+k_{n}\left(L_{12}^{(h)} Z^{n}+n l y\right)\right)$ \\
Calculer & $Z^{n+1}=\left(I d_{2}-k_{n} L_{22}^{(h)}\right)^{-1}\left(Z^{n}+k_{n}\left(L_{21}^{(h)} Y^{n+1}+n l z\right)\right)$ \\
\hline
\end{tabular}

\subsubsection{Résultats numériques}

Les résultats numériques présentés ici ont été comparés avec ceux de Lin Bo Zhang [Zha87], obtenus d'une part, avec une discrétisation spatiale par différences finies d'ordre deux et, d'autre part, par une méthode spectrale en Fourier. 


\section{Influence de l'ordre des schémas en espace}

On observe numériquement que le schéma semi-implicite en $Y-Z$ est d'autant plus stable que l'ordre d'interpolation est élevé, c'est-à-dire que les composantes en $Z$ sont petites ; c'est en fait caractéristique de l'approche $Y$ - $Z$ dès que le système est dissipatif. Cette influence est bien sûr renforcée si le schéma en espace n'est pas suffisamment précis ou si le pas de temps n'est pas assez petit : la perte de précision, typiquement l'affaissement de la "bosse" centrale du profil de flamme, survient pour des valeurs de Z pas assez "petites". C'est un indicateur pour développer des schémas adaptatifs pour cette équation. Par exemple, il a fallu prendre pour $N_{j}=64(32$ pts sur la grille grossière) et $\ell=50$, des inconnues d'ordre 8 , le pas de temps étant le même que celui de la méthode classique, à savoir $\delta t=0,01$.

\section{Validation des résultats}

Les comparaisons que nous faisons avec les résultats de Lin-Bo Zhang portent sur l'évolution en temps de la moyenne et sur l'évolution de la configuration du front de flamme à des temps donnés, étant évidemment partis de la même condition initiale : $u(x, 0)=\cos \left(\frac{2 \pi x}{\ell}\right)$.

Le code en Fourier fournit exactement les mêmes résultats que ceux de Zhang, il peut donc servir de référence. C'est classique, mais particulièrement valable pour $\mathrm{KS}$, le nombre de points de discrétisation nécessaire pour bien représenter la solution est très fortement lié à la précision avec laquelle les opérateurs différentiels sont approchés : les schémas aux différences finies d'ordre élevés, tels les schémas compacts utilisés ici, permettent d'obtenir des résultats comparables avec ceux en Fourier en utilisant le même nombre de points.

Les résultats présentés ci-dessous ont été obtenus avec une discrétisation en espace d'ordre quatre et pour les valeurs de $\ell, N_{j}$ (nombre de sous-intervalles) et $\delta t$ suivantes :

- $\ell=10 ; N_{j}=16, \delta t=0,1$

- $\ell=20 ; N_{j}=32, \delta t=0,1$

- $\ell=50 ; N_{j}=64, \delta t=0,01$ (pour Euler et Euler Y-Z), $\delta t=0,1$ pour (RK43)

- $\ell=100 ; N_{j}=128, \delta t=0,005$

pour un jeu de paramètres donné $N_{j}$, le nombre de points est le même que celui utilisé dans le code Fourier.

Pour différents ordres de discrétisation en espace par différences finies, nous comparons les résultats fournis par les schémas (classiques) à une grille (KS nodal noté aussi KSE) et par ceux à deux grilles (KS en Y-Z noté aussi KSE-YZ). Nous examinons en particulier l'évolution de la moyenne pour différentes tailles $\ell$ du domaine (Figure 7) mais aussi du front de flamme (voir Figures 8-9-10-11 et [Zha87]).

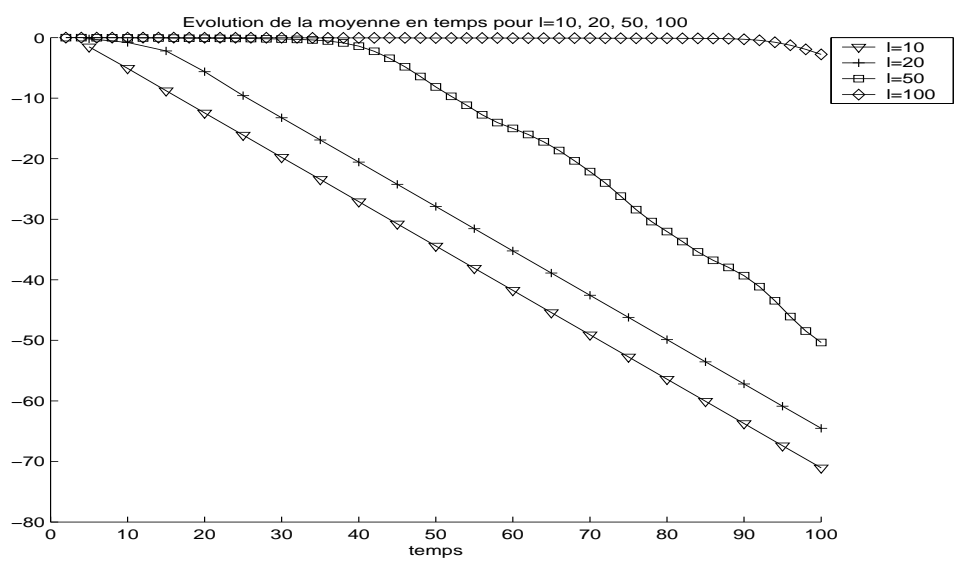

Figure 7. Moyenne en temps de la solution pour $\ell=10,20,50,100$ 

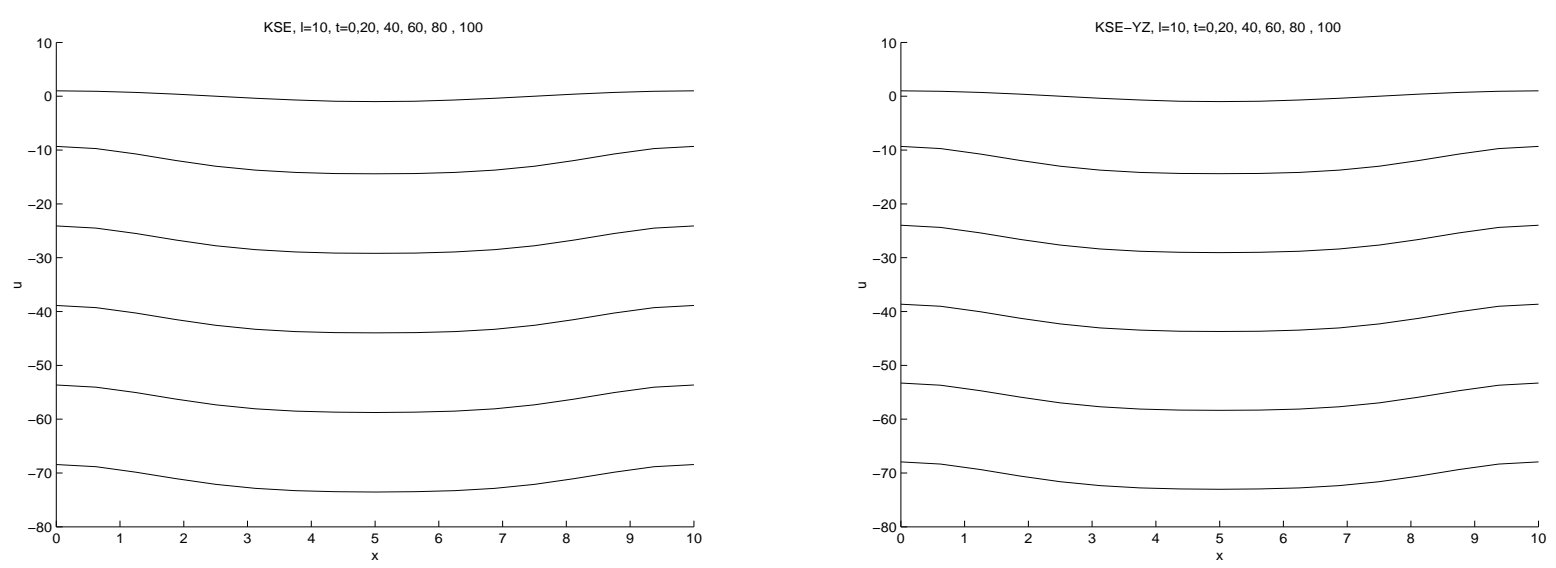

Figure 8. Front de flamme au temps 0, 20, 40, 60, 80, 100 pour $\ell=10, \delta t=0,01, N_{j}=64$. a) Euler classique, b) Euler Y-Z (IU2)
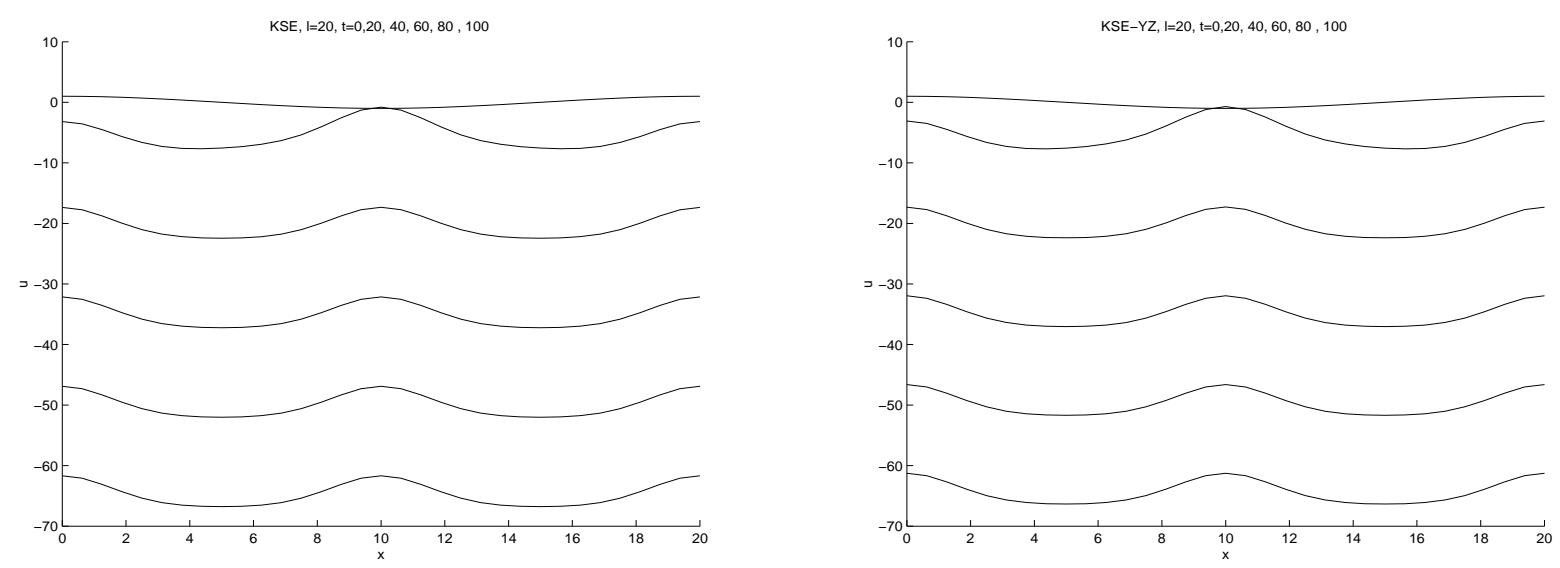

Figure 9. Front de flamme au temps 0, 20, 40, 60, 80, 100 pour $\ell=20, \delta t=0,01, N_{j}=64$. a) Euler classique, b) Euler Y-Z (IU4)

On représente maintenant l'évolution du front de flamme pour différents jeux de paramètres, pour les schémas classiques et pour ceux en Y-Z. Pour $\ell=50$ et $N_{j}=64$ (Figure 10), on observe un léger affaissement (oscillation) de la bosse centrale alors qu'elle devrait-être parfaitement plane, cela est vrai pour KS nodal comme pour KS en Y-Z. Ce défaut disparaît en prenant plus de points ou en augmentant l'ordre de discrétisation en espace. Pour $\ell=100$ (Figure 11) et $N_{j}=128$ les résultats de KS nodal et KS en Y-Z sont comparables. Concernant la rapidité d'execution, le code en $Y-Z$ nécessite d'inverser à chaque étape 2 matrices de taille $N_{j} / 2$ au lieu d'une matrice de taille $N_{j}$ pour KS nodal.

\section{Ordre des IU}

Nous comparons enfin les résultats pour $\ell=50$ et $N_{j}=64$ en prenant des discrétisations d'ordre élevé en espace (schémas compacts (CS)) et en faisant varier l'ordre des inconnues incrémentales. Il apparaît clairement que l'ordre de grandeur des inconnues incrémentales influe directement sur la précision du schéma. Les résultats du schéma en Y-Z présentés en Figure 12 et Figure 13 sont comparables à ceux du schéma classique dès que sont 

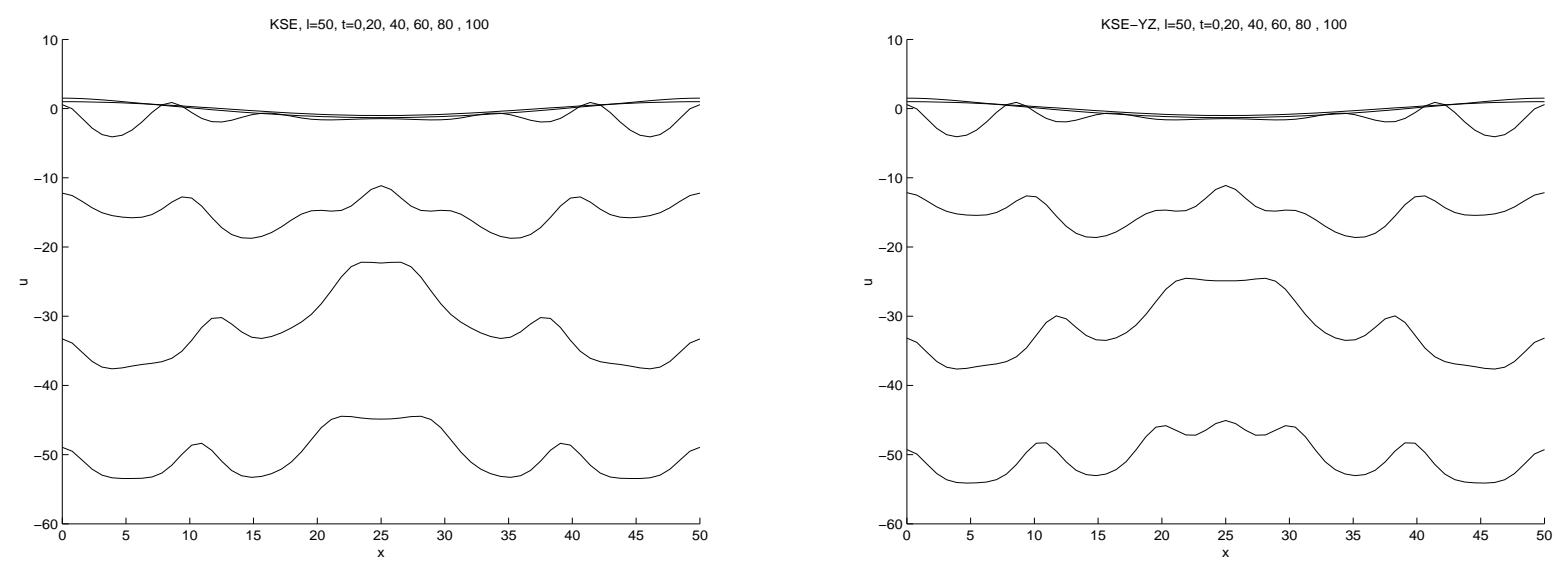

Figure 10. Front de flamme au temps 0, 20, 40, 60, 80, 100 pour $\ell=50, \delta t=0,01, N_{j}=64$.

a) Euler classique, b) Euler Y-Z (IU8)
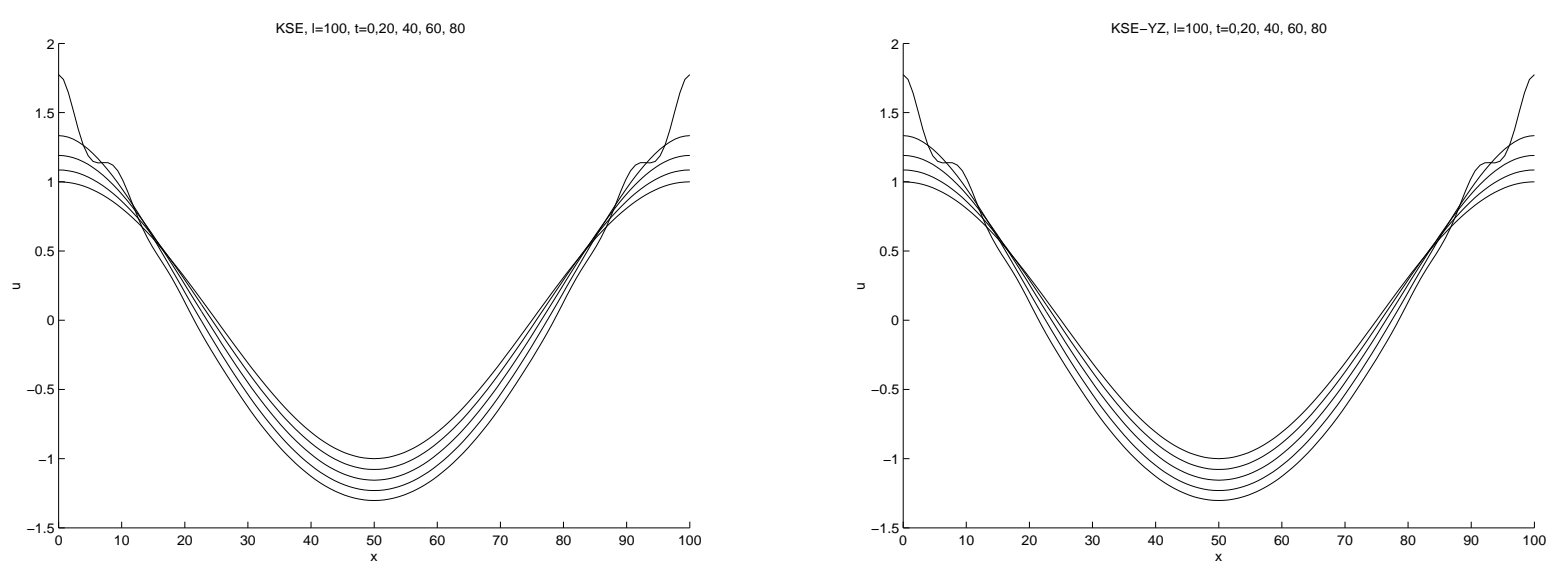

Figure 11. Front de flamme au temps 0, 20, 40, 60, 80 pour $\ell=100, \delta t=0,01, N_{j}=64$. a) Euler classique, b) Euler Y-Z (IU8)

utilisées des inconnues incrémentales d'ordre 6 . 

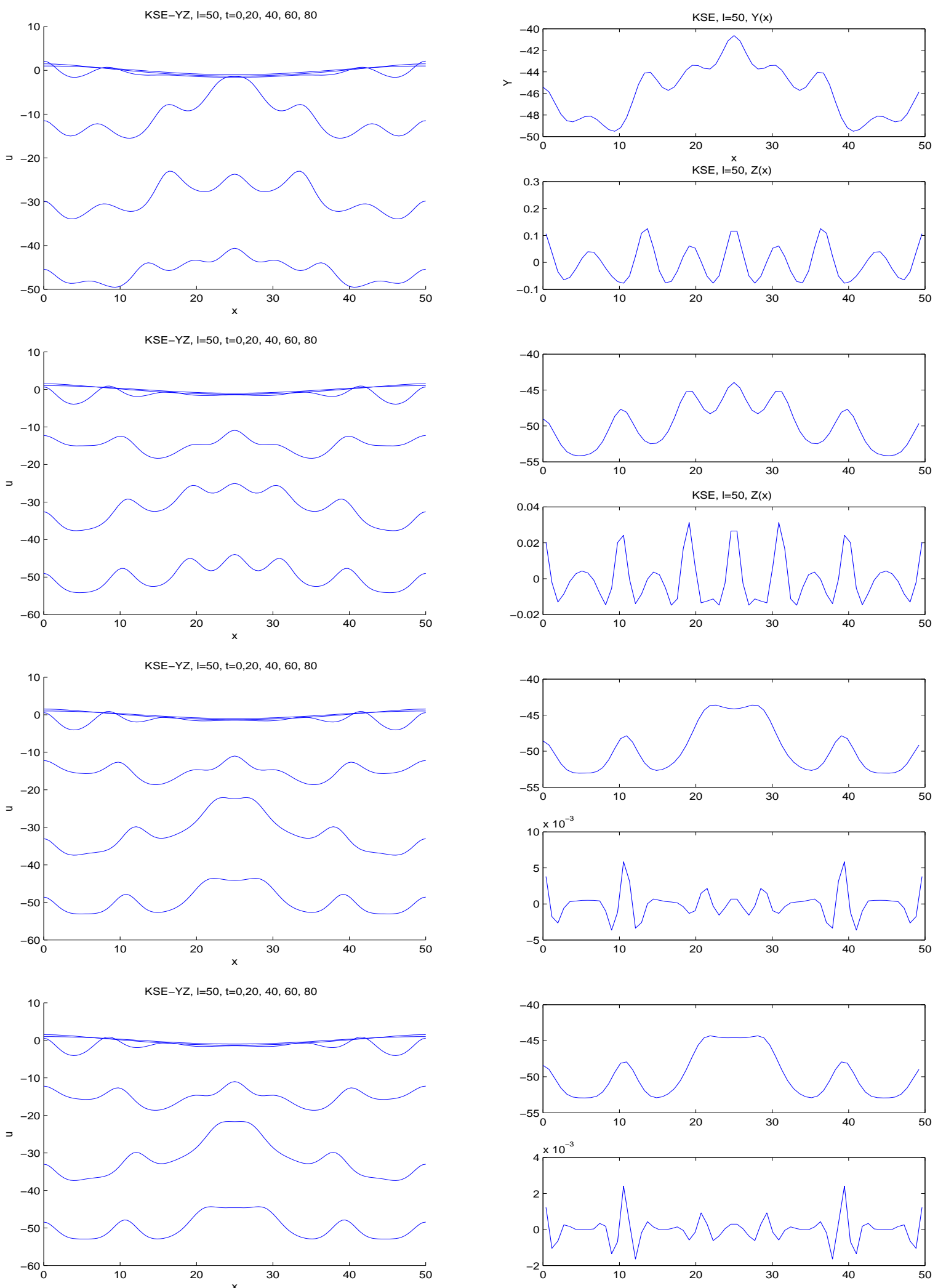

Figure 12. Front de flamme au temps 0, 20, 40, 60, 80 pour $\ell=50, \delta t=0,005, N_{j}=64$. Discrétisation en espace : CS 4, a) IU2, b) IU4, c) IU6, d) IU8. 

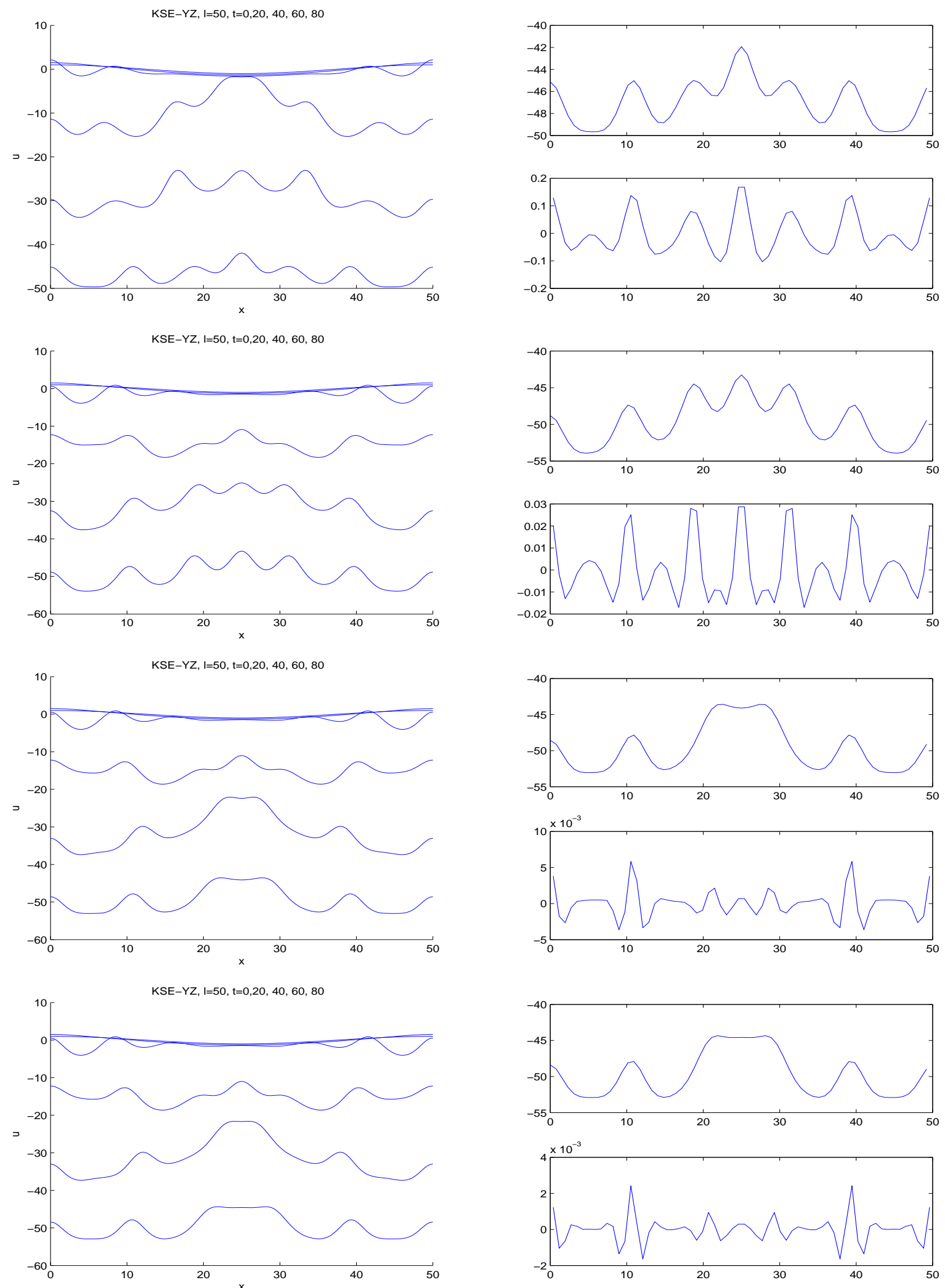

Figure 13. Front de flamme au temps 0, 20, 40, 60, 80 pour $\ell=50, \delta t=0,005, N_{j}=64$. Discrétisation en espace : CS 6, a) IU2, b) IU4, c) IU6, d) IU8. 


\section{Conclusion}

A travers ce travail, nous avons essayé de mettre en œuvre, en les adaptant, des méthodes multiniveaux fondées sur les idées de GNL. Il ressort de nos simulations que le manque de régularisation, donc de transfert d'énergie des hauts vers les bas modes de Fourier, pour KdV, ne permet pas d'adapter directement la stratégie utilisée pour les équations dissipatives. A contrario, l'équation de Kuramoto Sivashinki, par ses propriétés dissipatives rend possible une telle adaptation, ce qui permet d'appliquer avec succès les techniques multiniveaux. Notons tout de même que l'analyse des solutions numériques par des bases hiérarchiques, ondelettes ou inconnues incrémentales, se présente comme un outil pour développer des maillages adaptatifs, pour capter par exemple une solution trẤls localisée en espace. Elles peuvent également être mises en ouvre pour filtrer certaines fréquences (seuillage).

Les propriétés de régularisation semblent donc bien être nécessaires à l'application d'une approche multiniveaux de type GNL. Il serait néanmoins intéressant de considérer les équations faiblement amorties, telles Schrödinger ou $\mathrm{KdV}\left[\mathrm{AMC}^{+}\right.$08, AMCC08], pour lesquelles les solutions sont régularisées asymptotiquement en temps ; cela a été établi par exemple pour KdV faiblement amorti, [Ghi94, Gou00, GR02]. Ceci se présente comme une situation intermédiaire entre le cas dissipatif et celui dispersif.

\section{REMERCIEMENTS}

Ce travail a été réalisé dans le cadre du projet CNRS/DGRST, Action d'échanges 2003, code 03/R 1503, "Simulation multi-échelle et analyse mathématique des équations d'ondes dispersives". Il a également bénéficié du support du programme Méditerranée 3+3 MASOH (Modélisation Analyse Simulation Ondes Hydrodynamiques) de l'INRIA. Les auteurs rémercient les rapporteurs anonymes pour leurs observations et leurs critiques constructives qui ont permis d'améliorer la présentation de ce travail.

\section{REFERENCES}

$\left[\mathrm{AMC}^{+} 08\right]$ M. Abounouh, H. Al Moatassime, J-P. Chehab, S. Dumont, and O. Goubet. Discrete schrodinger equations and dissipative dynamical systems. Communications on Pure and Applied Analysis, 7(2):211-227, 2008.

[AMCC08] M. Abounouh, H. Al Moatassime, C. Calgaro, and J-P. Chehab. Global attractor for a discrete forced and damped kdv equation. En préparation, 2008.

[BDK86] J. Bona, V. Dougalis, and O. Karakashian. Fully discrete galerkin methods for the korteweg-de vries equation. Comput. Math. Appl., Part A 12:859-884, 1986.

[BDKM91] J. L. Bona, V. A. Dougalis, O. A. Karakashian, and W. R. McKinney. Fully-discrete methods with grid refinement for the generalized Korteweg-de Vries equation. In Viscous profiles and numerical methods for shock waves (Raleigh, NC, 1990), pages 1-11. SIAM, Philadelphia, PA, 1991.

[BDKM95] J. Bona, V. Dougalis, O. Karakashian, and W. McKinney. Conservative, high-order numerical schemes for the generalized korteweg de vries equation. Philos. Trans. R. Soc. Lond., 1695:107-164, 1995.

[BDKM96] J. L. Bona, V. A. Dougalis, O. A. Karakashian, and W. R. McKinney. Numerical simulation of singular solutions of the generalized Korteweg-de Vries equation. In Mathematical problems in the theory of water waves (Luminy, 1995), volume 200 of Contemp. Math., pages 17-29. Amer. Math. Soc., Providence, RI, 1996.

[BK04] J. L. Bona and H. Kalisch. Singularity formation in the generalized Benjamin-Ono equation. Discrete Contin. Dyn. Syst., 11(1):27-45, 2004.

[CC03] J.-P. Chehab and B. Costa. Multiparameter schemes for evolutive pdes. Numerical Algorithms, 34:245-257, 2003.

[CC04] J.-P. Chehab and B. Costa. Time explicit schemes and spatial finite differences splittings. J. Scient. Computing, 20(2):159-189, 2004.

[CCLZ08] C. Calgaro, J.-P. Chehab, J. Laminie, and E. Zahrouni. Schémas multiniveaux pour les équations d'ondes en dimension un. Technical Report 00271154, INRIA, 2008.

[CDGT01] B. Costa, L. Dettori, D. Gottlieb, and R. Temam. Time marching techniques for the nonlinear galerkin method. SIAM J. SC. comp., 23:46-65, 2001.

[CDL98] C. Calgaro, A. Debussche, and J. Laminie. On a multilevel approach for the two dimensional Navier-Stokes equations with finite elements. Int. J. for Num. Meth. in Fluids, 27:241-258, 1998.

[Che98] Jean-Paul Chehab. Incremental unknowns method and compact schemes. RAIRO Modél. Math. Anal. Numér., 32(1):51-83, 1998. 
[CL08] C. Calgaro and J. Laminie. Numerical studies of a dynamical multilevel scheme for the navier stokes problem in the context of a domain decomposition method. Technical report, en préparation, 2008.

[CLT97] C. Calgaro, J. Laminie, and R. Temam. Dynamic multilevel schemes for the solution of evolution equations by hierarchical finite element discretization. Applied Numer. Math., 23:403-442, 1997.

[CM84] M. Crouzeix and A. L. Mignot. Analyse numérique des équations différentielles. Collection Mathématiques Appliquées pour la Maîtrise. Masson, Paris, 1984.

[CM98] J.-P. Chehab and A. Miranville. Incremental unknowns on nonuniform meshes. RAIRO Modél. Math. Anal. Numér., 32(5):539-577, 1998.

[CT91a] M. Chen and R Temam. The incremental unknown method 1. Applied Mathematics Letters, 4(1-3):73-76, 1991.

[CT91b] M. Chen and R Temam. The incremental unknown method 2. Applied Mathematics Letters, 4(3):77-80, 1991.

[Dau93] I. Daubechies. Ten Lectures on Wavelets. SIAM, Philadelphia, 1993.

[DD89] G. Deslauriers and S. Dubuc. Symmetric iterative interpolation processes. Constructive Approximation, 5(1):49-68, 1989.

[DDT95] A. Debussche, T. Dubois, and R. Temam. The nonlinear garlerkin method : A multiscale method applied to the simulation of homogeneous turbulent flows. Theoretical and Computational Fluid Dynamics, 7:279-15, 1995.

[Dem96] Jean-Pierre Demailly. Analyse numérique et équations différentielles. Collection Grenoble Sciences. Presses Universitaires de Grenoble, Grenoble, 1996.

[DFP81] M. Delfour, M. Fortin, and G. Payre. Finite difference solution of a nonlinear schrödinger equation. JCP, 44:277-288, 1981.

[DJT98] T. Dubois, F. Jauberteau, and R. Temam. Dynamic multilevel methods and the numerical simulation of turbulence. Cambridge University Press, 1998.

[DLZ05] A. Debussche, J. Laminie, and E. Zahrouni. A dynamical multilevel scheme for the burgers equation: wavelet and hierarchical finite element. J. Sc. Comput., 25(3):445-497, 2005.

[Don92] David L. Donoho. Interpolating wavelet transforms. Technical report, Stanford University, 1992. Publications of the Dep. of Statistics.

[Dri02] T. A. Driscoll. A composite runge-kutta method for the spectral solution of semilinear pde. J. Comp. Phys., 182:357367, 2002.

[DSS00] A. Durán and J.M. Sanz-Serna. The numerical integration of a relative equilibrium solutions. the nonlinear schrödinger equation. IMA J. Num. Anal., 20:235-261, 2000.

[Ghi88] J.M. Ghidaglia. Weakly damped forced korteweg-de vries equations behave as a finite dimensional dynamical system in the long time. J. Diff. Eq., 74:369-390, 1988.

[Ghi94] J.M. Ghidaglia. A note on the strong convergence towards attractors for damped forced kdv equations. J. Diff. Eq., 110:356-359, 1994.

[Gou93] O. Goubet. Nonlinear galerkin methods using hierarchical almost-orthogonal finite elements bases. Nonlinear Anal., Theory Methods Appl., 20(3):223-247, 1993.

[Gou00] O. Goubet. Asymptotic smoothing effect for weakly damped forced korteweg-de vries equations. Discrete Contin. Dynam. Systems, 6(3):625-644, 2000.

[GR02] O. Goubet and R. Rosa. Asymptotic smoothing and the global attractor of a weakly damped kdv equation on the real line. J. Differential Equations, 185(1):25-53, 2002.

[JRT01] M. Jolly, R. Rosa, and R. Temam. Accurate computations on inertial manifolds. Siam J. Sc. Comput., 22(6):2216-2238, 2001.

[Lel92] Sanjiva K. Lele. Compact finite difference schemes with spectral-like resolution. J. Comput. Phys., 103(1):16-42, 1992.

[Mal00] S. Mallat. Une exploration des signaux en ondelettes. Ecole Polytechnique, Paris, 2000.

[MT89] M. Marion and R. Temam. Nonlinear galerkin methods. SIAM J. Numer. Anal., 26:1139-1157, 1989.

[MT90] M. Marion and R. Temam. Nonlinear galerkin methods : the finite element case. Numer. Math., 57:205-226, 1990.

[Pas92] F. Pascal. Méthodes de Galerkin non lináires en discrétisation par éléments finis et pseudo-spectrale. Application à la mécanique des fluides. Thèse, Université Paris Sud, Mathématique, 1992.

[Pou98] F. Pouit. Etude de schémas numériques multiniveaux utilisant les inconnues incrémentales dans le cas des différences finies : application à la mécanique des fluides. Thèse, Université Paris Sud, Mathématique, 1998.

[Tem97] R. Temam. Infinite Dimensional Dynamical Systems in Mechanics and Physics. Number 68 in Applied Mathematical Science. Springer Verlag, 1997.

[Zha87] Lin-Bo Zhang. Un schéma de semi-discrétisation en temps pour des systèmes différentiels discrétisés en espace par la méthode de Fourier. Résolution numérique des équations de Navier-Stokes stationnaires par la méthode multigrille. Thèse, Université Paris Sud, Mathématique, 1987. 